\title{
Frequency spectra of horizontal winds in the mesosphere and lower thermosphere region from multistatic specular meteor radar observations during the SIMONe 2018 campaign
}

\section{Harikrishnan Charuvil Asokan ( $\nabla$ hari@iap-kborn.de )}

Leibniz Institute of Atmospheric Physics: Leibniz-Institut fur Atmospharenphysik eV an der Universitat Rostock https://orcid.org/0000-0002-3628-5568

\section{Jorge L Chau}

Leibniz Institute of Atmospheric Physics: Leibniz-Institut fur Atmospharenphysik eV an der Universitat Rostock

\section{Raffaele Marino}

École Centrale de Lyon: Ecole Centrale de Lyon

\section{Juha Vierinen}

University of Tromso: UiT Norges arktiske universitet

\section{Fabio Vargas}

University of Illinois at Urbana-Champaign

\section{Juan Miguel Urco}

University of Illinois at Urbana-Champaign

\section{Matthias Clahsen}

Leibniz Institute of Atmospheric Physics: Leibniz-Institut fur Atmospharenphysik eV an der Universitat Rostock

Christoph Jacobi

Leipzig University: Universitat Leipzig

\section{Full paper}

Keywords: Frequency Spectra, Mesosphere and Lower Thermosphere, Specular Meteor Radar, Horizontal winds, Wind field Correlation Function Inversion, SIMONe 2018

Posted Date: July 13th, 2021

DOl: https://doi.org/10.21203/rs.3.rs-697043/v1

License: (c) (i) This work is licensed under a Creative Commons Attribution 4.0 International License. 



\section{. Frequency spectra of horizontal winds in the mesosphere and}

\section{2 lower thermosphere region from multistatic specular meteor 3 radar observations during the SIMONe 2018 campaign}

$4 \quad$ Harikrishnan Charuvil Asokan, Leibniz-Institute of Atmospheric Physics at the Rostock University, Kühlungsborn, Germany, and Laboratoire de Mécanique des Fluides et d'Acoustique, CNRS, École Centrale de Lyon, Université Claude Bernard Lyon 1, INSA de Lyon, Écully, France, hari@iap-kborn.de

Jorge L. Chau, Leibniz-Institute of Atmospheric Physics at the Rostock University, Kühlungsborn, Germany, chau@iap-kborn.de

Raffaele Marino, Laboratoire de Mécanique des Fluides et d'Acoustique, CNRS, École Centrale de Lyon, Université Claude Bernard Lyon 1, INSA de Lyon, Écully, France, raffaele.marino@ec-lyon.fr Juha Vierinen, Department of Physics and Technology, University of Tromsø, The Arctic University of Norway, Tromsø, Norway, juha-pekka.vierinen@uit.no Fabio Vargas, Department of Electrical and Computer Engineering, University of Illinois at Urbana-Champaign, Urbana, IL, USA, 61801, fvargas@illinois.edu Juan Miguel Urco, Department of Electrical and Computer Engineering, University of Illinois at Urbana-Champaign, Urbana, IL, USA, 61801, mcordero@illinois.edu Matthias Clahsen, Leibniz-Institute of Atmospheric Physics at the Rostock University, Kühlungsborn, Germany, clahsen@iap-kborn.de

Christoph Jacobi, Institute for Meteorology, Universität Leipzig, Stephanstr. 3, 04103 Leipzig, Germany, jacobi@uni-leipzig.de 


\section{Abstract}

7 In recent years, multistatic specular meteor radars (SMRs) have been introduced to study the

8 Mesosphere and Lower Thermosphere (MLT) dynamics with increasing spatial and temporal resolution.

9 In this paper, frequency spectra of MLT horizontal winds are explored through observations from a 10 campaign using the SIMONe (Spread-spectrum Interferometric Multistatic meteor radar Observing ${ }_{11}$ Network) approach conducted in northern Germany in 2018 (hereafter SIMONe 2018). The seven-day SIMONe 2018 comprised of fourteen multistatic SMR links and allows to build a substantial database of 13 specular meteor trail events, collecting more than one hundred thousand detections per day within a 14 geographic area of $\sim 500 \mathrm{~km} \times 500 \mathrm{~km}$. We have implemented two methods to obtain the frequency spectra of the horizontal wind components: (1) Mean Wind Estimation (MWE) and (2) Wind field 16 Correlation Function Inversion (WCFI), which utilizes the mean and the covariances of the line of sight 17 velocities, respectively. Monte Carlo simulations of a gravity wave spectral model were implemented to validate and compare both methods. The simulation analyses suggest that the WCFI helps to capture the energy of smaller-scale wind fluctuations than those capture with MWE. Characterization of the spectral slope of the horizontal wind at different MLT altitudes has been conducted on the SIMONe 2018, and it provides evidence that gravity waves with periods smaller than seven hours and greater than two hours dominate with horizontal structures significantly larger than $500 \mathrm{~km}$. These waves might be associated with secondary gravity waves during this observational campaign. In the future,

24 these analyses can be extended to understand the significance of small-scale fluctuations in the MLT, 25 which were not possible with conventional MWE methods.

\section{Keywords}

${ }_{27}$ Frequency Spectra, Mesosphere and Lower Thermosphere, Specular Meteor Radar, Horizontal winds, ${ }_{28}$ Wind field Correlation Function Inversion, SIMONe 2018

\section{Introduction}

30 The mesosphere and lower thermosphere (MLT) is an extremely dynamic region of the atmosphere located ${ }_{31}$ between 60 and $110 \mathrm{~km}$ above the Earth's surface. At middle and high latitudes, the region is cold in 
summer, but relatively warm in winter, and the lowest temperature in the Earth's atmosphere is found at 33 the polar summer mesopause. The rich dynamical activity in the MLT region is mostly attributed to the 34 propagation and breaking of gravity waves $(\mathrm{GWs})$, the creation of turbulent structures, the emergence 35 of atmospheric tides and planetary waves. The deposition of energy and momentum in the MLT region 36 by atmospheric GWs plays an essential role in the middle atmospheric dynamics (Lindzen 1981; Holton 37 1983; Vincent and Reid 1983; Yiğit and Medvedev 2015).

38 Continuous in-situ observations of the MLT are challenging. Indeed meteorological balloons and aircraft 39 cannot fly as high as MLT altitudes, while satellites orbit above the MLT and cannot make in situ 40 measurements. Instruments on sounding rockets can be used to investigate the MLT; however, they are ${ }_{41}$ brief, infrequent, and expensive. In the last few decades, the community was able to answer scientific 42 questions by performing continuous observations of the MLT using different ground-based remote sensing 43 technologies such as lidars, airglow imagers and radars. Lidar measurements of temperature, density 44 and wind measurements are used to investigate GWs in the stratosphere and mesosphere (e.g., Chanin 45 and Hauchecorne 1981; Gardner and Voelz 1987; Alexander et al. 2011; Baumgarten 2010; Baumgarten 46 et al. 2015; Chen et al. 2016; Kaifler et al. 2017; Strelnikova et al. 2020). Another technique to observe 47 mesospheric GWs is the imaging of airglow emission layers, which provides information about spatial ${ }_{48}$ and temporal characteristics of the GWs (e.g., Swenson and Mende 1994; Medeiros et al. 2003; Espy et al. 2004; Vargas et al. 2007, 2016; Wüst et al. 2017; Taylor et al. 2019; Vargas 2019). Mesosphere50 stratosphere-troposphere (MST), medium frequency (MF), incoherent scatter and specular meteor radars ${ }_{51}$ (SMRs) are some of the various types of radars utilized to estimate mean winds and correlations, from which it is possible to examine GWs and turbulence in the MLT region (e.g., Gage and Balsley 1984; 53 Reid 1990; Nakamura et al. 1993; Zhou 2000; Antonita et al. 2008; Fritts et al. 2010; Placke et al. 2011a,b, 54 2015; Yasui et al. 2016; Tsutsumi et al. 2017; Reid et al. 2018).

SMRs are a viable option to study the spatial and temporal evolution of wind velocity fields in the MLT,

56 the primary constraint being the limited number of meteors crossing the observed region per unit time ${ }_{57}$ and the viewing angle diversity. These radars use radio scattering from meteor trails to estimate the MLT winds and other parameters.

59 In this paper, we analyse data obtained from an ambitious ground-based meteor radar observational campaign that uses the SIMONe (Spread-spectrum Interferometric Multistatic meteor radar Observing ${ }_{61}$ Network) approach (Chau et al. 2019). This campaign, called SIMONe 2018, was conducted in northern 
71 of a typical monostatic SMR

72 Global circulation models (GCMs) and observational studies underlined the feedback of the small scale

Germany for seven consecutive days from November 2 to November 9, 2018. SIMONe 2018 benefited from the implementation of the MMARIA (Multistatic, multi-frequency Agile Radar Investigations of the Atmosphere) concept, which is a multistatic and multi-frequency approach for SMRs to improve the MLT wind measurements (Stober and Chau 2015), combined with a new radar signal processing technique for coded continuous wave meteor radar transmissions (Vierinen et al. 2016), the use of multiple transmitters and multiple receivers for radar interferometry (Urco et al. 2018), and compressed sensing (Urco et al. 2019). The campaign helped to build a unique data set that collected more than one hundred thousand specular meteor detections per day across a large geographic area $(500 \mathrm{~km} \times 500 \mathrm{~km})$ in the MLT. Therefore, this data set contains observations of meteor trails that are 10 to 20 times greater than those GWs on the general circulation of the middle atmosphere (Alexander et al. 2010). GCMs generally do not resolve the small scale waves with horizontal wavelengths shorter than $1000 \mathrm{~km}$ (Kim et al. 2003; Geller et al. 2013). Hence GCMs include the influence of these GWs by using various parametrization schemes (McLandress 1998). Liu et al. (2014) and Liu (2019) suggested that small mesoscale GWs may contribute significantly to the momentum budget of the middle and upper atmosphere. Therefore, detailed observations of smaller-scale GWs are needed to constrain these parameterizations.

Together with studies based on global models, in recent years the role of GWs in stratified geophysical flows has been widely addressed also from a fundamental point of view (Marino et al. 2013, 2014), by means of theoretical modeling and high-resolution direct numerical simulations (DNS). In particular, state of the art DNS allow to access a parameter space compatible with that of the MLT, without resorting to the use of models for the small scales. This makes the MLT a unique natural laboratory in which is possible to test turbulence theories and get insights on the mechanisms underlying the dynamics of high-Reynolds number stratified flows as it results from the interplay of GWs and turbulent motions (Marino et al. 2015b; Herbert et al. 2016; Feraco et al. 2018, 2021). While DNS are able to provide the point-wise prognostic fields of turbulent flows of geophysical interest (Rosenberg et al. 2015; Marino et al. 2015a; Pouquet et al. 2017), with a very high spatial resolution, computational capability of modern supercomputers does not allow to run DNS on large grids and over a large number of characteristic turbulence and wave times, such as the eddy turnover time or the time scales associated to inertia-gravity waves (Marino et al. 2013). This makes it of critical importance to combine fundamental analytical and numerical approaches with 
state of the art time-continuous observations (overextended spatial and temporal domains) such as those obtained in the frame of SIMONe 2018.

The power spectra of the MLT winds using observations are discussed in a few studies in the literature (Gage and Balsley 1984; Balsley and Garello 1985; Weinstock 1996; Manson et al. 1999). Recently, Sato et al. (2017) presented the power and momentum flux spectra of neutral wind components in a frequency range from $(8 \mathrm{~min})^{-1}$ to $(20 \text { days })^{-1}$ using MST radar observations of polar mesospheric summer echoes. However, spectral studies of the MLT using SMRs are discussed seldom in the literature. This work aims to use SMRs to characterize fundamental fluid dynamics properties of the MLT and advance our understanding of the vertical propagation of GWs and their possible role on the energy budget of the upper atmosphere. This will be achieved in the frame of the proposed study by means of classical and newly developed methodologies to compute the horizontal wind frequency spectra from the multistatic SMR wind signals coming from the MLT using both first-order and second-order line of sight velocity statistics. In particular, the frequency spectra on different horizontal scales for horizontal wind fluctuations will be examined. We also used synthetic data based on a GW spectral model to understand and verify the spectral slope variations of the two methods. The manuscript thus starts with an overview of the SIMONe 2018 campaign, including a summary of the observations. Then the details of the two frequency-spectra methods implementing first and second-order statistics of line-of-sight velocities are presented. Monte Carlo simulations of a GW spectral model are described and shown in methodology section to validate and compare the two methods. The comparison of the results with the two methods and the possible connection to secondary GWs is discussed before the conclusions.

\section{Campaign Overview}

In November 2018 we conducted a seven-day multistatic specular meteor radar campaign called SIMONe 2018, comprising of fourteen SMR links. Six links were obtained with existing pulsed systems, while the remaining eight links resulted from implementing a SIMONe approach, i.e., coded continuous wave $(\mathrm{CW})$ transmissions and MIMO technology (Chau et al. 2019).

The pulsed transmitters were located at Juliusruh $\left(54.63^{\circ} \mathrm{N}, 13.37^{\circ} \mathrm{E}\right)$ and Collm $\left(51.31^{\circ} \mathrm{N}, 13.00^{\circ} \mathrm{E}\right)$ operating at $32.55 \mathrm{MHz}$ and $36.2 \mathrm{MHz}$, respectively. Both systems operated with $1.6 \mathrm{~ms}$ interpulse period (IPP), seven-Barker code with $10 \mu$ s baud lengths. The Collm transmitter is delayed $540 \mu$ s with respect to Juliusruh, to keep the same sampling window at the receiving station and shift the region of 
maximum detections away from the sampling edges.

The coded-CW transmitter site was located at Kühlungsborn $\left(54.12^{\circ} \mathrm{N}, 11.77^{\circ} \mathrm{W}\right)$ and consisted of five antennas, each of them transmitting a different pseudo random binary phase code of 1000 bauds each. The baud length was $10 \mu$ s, i.e., the sequence was repeated every $10 \mathrm{~ms}$, allowing an unambiguous total range of $6000 \mathrm{~km}$. The antennas were configured in a pentagon configuration. Similar pentagon configurations have been used by Younger and Reid (2017) and Chau and Clahsen (2019) who have shown that a pentagon configuration present sidelobes with larger separation, lower amplitude and better symmetry than those obtained with the so-called Jones configuration (Jones et al. 1998) used in most monostatic SMRs.

On reception, six sites were used. In Table 1, we provide the relevant information for each site: location, reception mode and, reception type. In the case of pulse links, detection and identification of meteor events has been done following Hocking et al. (2001); Holdsworth et al. (2004). For the coded-CW links, depending on the number of antennas used on transmission or reception, they can be considered multi-input single-output (MISO) or single-input multiple-output (SIMO), and therefore either the angleof-departure (AOD) or angle-of-arrival (AOA) is estimated, respectively. Specific details of the SIMONe methodology are given by Chau et al. (2019).

We measured the location, Bragg vectors, and Doppler frequencies and their statistical uncertainties of each specular meteor echoes for all the links during the campaign. Figure 1 shows a summary of the campaign. In the upper row we show: (a) a 2D histogram of detections as function of latitude and longitude, (b) a height histogram for all detections, (c) height histograms for each link, (d) a 2D histogram as function of height and log of inverse decay time. The second and third rows (e,f) show the mean zonal and meridional velocities using all links, while the bottom row $(\mathrm{g})$ shows the hourly detections for each link for the whole campaign. Although we operated the systems for nearly 10 days, in this work we focused only on seven days, when the majority of links were operational. Given the success of the campaign, operational versions using the SIMONe approach were installed in central Peru and southern Argentina in September 2019 (Chau et al. 2021; Conte et al. 2021). 


\section{Methodology}

\section{Spectra through Mean Wind Estimation (MWE)}

SMRs use radio reflections from the specular meteor plasma trails, which drift with the mesospheric neutral wind to obtain the radial Doppler shift $\omega_{r}=\vec{k} \cdot \vec{v}(t, \vec{p})(\mathrm{rad} / \mathrm{s})$ of the wind at random locations where echoes are detected (Manning et al. 1950). Here, $\vec{k}=\left(k^{u}, k^{v}, k^{w}\right)$ is the Bragg wave vector of the radar, which is determined by the radar frequency and the observing geometry, and $\vec{v}(t, \vec{p})$ is the true wind velocity at any given position $\vec{p}$ and time $t$. Conventionally, the mean winds $(\vec{v})$ are estimated by leastsquare fitting of radial winds to mean wind velocities at a given altitude gate during a time bin, assuming the horizontal wind is homogeneous inside the observed volume (Hocking et al. 2001; Holdsworth et al. 2004).

Chau et al. (2017) relaxed the assumption of the homogeneity of the horizontal wind by applying a gradient approach that allowed estimating mean wind components and horizontal gradient terms. We utilized this gradient approach to estimate the mean zonal, meridional, and apparent vertical winds from the SIMONe 2018 campaign data. Similar approaches have been used previously using first-order Taylor expansion approximation of the horizontal wind field in the observed volume (e.g., Browning and Wexler 1968; Waldteufel and Corbin 1979; Burnside et al. 1981; Larsen et al. 1991; Conde and Smith 1998; Meriwether et al. 2008). However, most of these analyses focused on single station measurements and could not determine all the gradient terms independently. Chau et al. (2017) describe the gradient method and provide information about how the new approach reduces the contamination in vertical wind estimation due to a mean horizontal divergence.

Figure 2 shows the mean zonal wind (a), four-hour and four-km high pass filtered residual zonal wind (b), mean meridional wind (c), four-hour and four-km high pass filtered meridional wind (d), apparent vertical wind (e), and specular meteor counts obtained after averaging meteors within a $1 \mathrm{~km}$ height bin for every 30 minutes (f). SIMONe 2018 was able to reach a substantial number of specular meteor trails, collecting more than one hundred thousand detections per day, which helped us to estimate higher-resolution MLT winds compared to usual SMR observations. The estimated zonal and meridional wind velocities are then combined to determine the horizontal wind frequency spectra by Fourier transformation. To ease the comparison, the obtained spectra were averaged around three altitudes and are discussed later in the paper.

Note that we are labelling the vertical estimates as apparent vertical winds (w) since their relative large 
magnitudes lasting for a few hours are not expected. Hourly w magnitudes of a few meters per second are not uncommon (e.g., Larsen and Meriwether 2012; Lu et al. 2017), however their presence after averaging a relatively large horizontal area (250 km radius) is not expected. Such values would mean that horizontal structures with a few hundred of kilometer sizes would have a few meters per second vertical motions. Although traditional studies with SMRs have assumed "w=0", we have decided to include them in this work to share with the readers these intrigued results.

\section{Spectra through Wind Field Correlation Function Inversion (WCFI)}

Correlation of the mesospheric winds was first studied by Vincent and Reid (1983) using dual coplanar beam radar measurements. This technique was extended by Thorsen et al. (1997) and Hocking (2005) for MF radar systems with single vertical antenna beams and, all-sky monostatic SMRs, respectively. Vierinen et al. (2019) generalized the previous approaches for estimating wind correlations and described Wind field Correlation Function Inversion (WCFI) as a method to investigate stratified turbulence and GWs, which allows estimating the second-order statistical parameters of the real three-dimensional wind field using SMR observations. Each of the Doppler measurements obtained from the multistatic SMRs is a one-dimensional projection (line-of-sight) of the wind velocity vector sampled randomly in space and time. The WCFI method uses the products of such sparse measurements to estimate spatiotemporal correlation functions.

The product of these Doppler measurement pairs contains information about the wind field correlations. For each $\mathrm{N}$ measurements, we can form $\mathrm{N}(\mathrm{N}-1) / 2$ unique measurement pairs. Thus, if we take crossproduct of any two Doppler measurements, we will be able to estimate the correlation functions. The product of these measurement pairs can be written as a linear equation:

$$
y=A x+\zeta,
$$

where $y$ is a vector of cross product measurements, $A$ is the kernel matrix, $x$ is the vector of correlation functions, and $\zeta$ is a vector with measurement errors.

In equation $1, \zeta=\xi_{i} \xi_{j}$, where $\xi_{i}$ and $\xi_{j}$ are the random variables representing the errors in the Doppler frequency measurements and, $\zeta$ is a zero-mean random variable associated with the expected statistical uncertainty. So $\zeta$ has a symmetric distribution centred around zero when $i \neq j$. In the case of self pairs, the Doppler velocity measurements are correlated for the product $\xi_{i} \xi_{i}$. It will violate the assumption of the zero-mean random variable condition of cross product measurements. If we include self lags, this 
will produce a bias in the correlation function estimation. In order to reduce the uncertainties in the estimated parameters, the WCFI method does not consider self-product measurements. The expected values of the products of the random variables can be expressed as a correlation function which is a function of temporal and spatial displacement. $G_{u u}(\tau, \vec{s}), G_{v v}(\tau, \vec{s}), G_{w w}(\tau, \vec{s}), G_{u v}(\tau, \vec{s}), G_{u w}(\tau, \vec{s})$, and $G_{v w}(\tau, \vec{s})$ are the six unique combinations of wind components found due to the symmetry of the linear equation. The correlation function $G_{\alpha \beta}(\tau, \vec{s})$ is a function of spatial and temporal displacement $\tau=t_{i}-t_{j}$ and $\vec{s}=\left(\overrightarrow{p_{i}}-\overrightarrow{p_{j}}\right)$, respectively, where $t_{i}$ and $t_{j}, \overrightarrow{p_{i}}$ and $\overrightarrow{p_{j}}$ are the time of occurrences and locations of the Doppler measurements' pairs. A detailed description of the method and equations can be found in Vierinen et al. (2019).

The application of the WCFI method on any SMR data will utilize the averaging of these spatial and temporal displacements or lag measurements. Based on the selection or restriction of different lags, the method separates them into spatial and temporal correlations of the MLT winds. This flexibility of the method allows us to study the temporal correlation functions with different horizontal scales.

Since the WCFI approach considers the estimation of correlation functions as an over-determined inversion problem, the method requires an adequate number of lagged products within the desired temporal and spatial displacements. Formations of spatial and temporal lags, which satisfy this condition can be applied to get the correlation functions. For the case of spatial correlation of the wind velocity, correlation functions are determined at every desired horizontal lag length by restricting the temporal and vertical lag resolutions to constant separations.

Similarly, temporal correlation functions are estimated at every desired temporal lag by restricting the horizontal and vertical lag resolution to constant values. These correlation functions are related to the power spectra by the Wiener-Khinchin theorem through a Fourier transform relationship (Wiener 1930; Khintchine 1934). This study focuses on obtaining the spectra from such temporal correlation functions which are determined at different horizontal lag resolutions. Figure 3 shows an example of a temporal autocorrelation function of meridional wind and the corresponding frequency spectra, obtained after averaging over 87 to $93 \mathrm{~km}$ in altitude with 1800 seconds (30 min) temporal lag, $50 \mathrm{~km}$ horizontal lag and $1 \mathrm{~km}$ vertical lag resolutions. The zero-lag value of the temporal autocorrelation function is about 580 $\mathrm{m}^{2} \mathrm{~s}^{-2}$ and the mean tidal wind mode at 12 hour lag is about $250 \mathrm{~m}^{2} \mathrm{~s}^{-2}$. This analysis of a seven-day data window can give an idea about the partition of energy between tides and GWs. Based on the example analysis, it is found that $40-45 \%$ of the kinetic energy in the meridional wind is associated with tides 
254 hour $^{-1}$.

25

ren

and planetary waves, and 50-55\% is associated with GWs and stratified turbulence, which is depicted by a blue arrow in the left plot.

\section{Gravity wave spectral model simulation}

A GW spectral model simulation was used to interpret the spectra obtained from the MWE and WCFI methods from the real meteor data. The simulation consists of two parts; (a) simulation of the wind field based on a GW spectral model (Gardner et al. 1993), and (b) implementation of this simulation to the SIMONe 2018 campaign geometry, and spatial and temporal sampling.

The first part simulation is a superposition of monochromatic GWs in which the amplitudes of the GWs depend on vertical wavenumber and frequency. The amplitude of the GWs, $A(m, \omega)$ is a product of a vertical wavenumber spectrum $(F(m))$ and an angular frequency spectrum $(B(\omega))$. Equations for these spectra were adopted from Gardner et al. (1993).

$$
A(m, \omega)=F(m) B(\omega)
$$

where $m$ is the vertical wavenumber and $\omega$ is the angular frequency of the wave. The amplitude of the vertical wavenumber spectrum is given below.

$$
F(m)=2 \pi \alpha N^{2} \begin{cases}m_{*}^{-3}\left(\frac{m}{m_{*}}\right)^{s} & , m \leq m_{*} \\ m_{*}^{-3} & , m_{*} \leq m \leq m_{b} \\ m_{b}^{-3}\left(\frac{m_{b}}{m}\right)^{\frac{5}{3}} & , m_{b} \leq m\end{cases}
$$

where $m$ is the vertical wavenumber, $m_{*}$ is the wavenumber of the largest scale saturated wave, $m_{b}$ is the buoyancy wavenumber, $\alpha$ is a constant which accounts for superposition effects, $N$ is the buoyancy frequency. We adopt values for these parameters from Senft and Gardner (1991); Gardner et al. (1993), i.e, $\alpha=0.62, N=\left(\frac{2 \pi}{3 \times 10^{2}}\right) \mathrm{s}^{-1}, m_{*}=\left(\frac{2 \pi}{1 \times 10^{4}}\right) \mathrm{m}^{-1}, m_{b}=\left(\frac{2 \pi}{5 \times 10^{2}}\right) \mathrm{m}^{-1}$, and $s=2$.

The amplitude of the angular frequency spectra is given by,

$$
B(\omega)=\frac{p-1}{f}\left(\frac{f}{\omega}\right)^{p},
$$

and the simulation assumes that the frequency spectrum is proportional to $\omega^{-p}$ where $p=2$ and, $f=\left(\frac{2 \pi}{20}\right)$

255 In the case of the angular frequency spectrum, we used 282 different wave periods between 10 minutes and

${ }_{256} 8$ hours. In the wavenumber spectrum, 40 different vertical wavelengths between $1 \mathrm{~km}$ and $20 \mathrm{~km}$ were ${ }^{257}$ used. In both cases, angular frequencies and wavenumbers were sampled uniformly. Thus, the combined 
2-D spectra became a superposition of more than $10000(\approx 282 \times 40)$ monochromatic GWs.

Each meteor measurement in the SIMONe 2018 data gives information mainly about its time of occurrence, its position in latitude, longitude and altitude from the ground, Doppler frequency of the meteor, and Bragg vector $(\vec{k})$. Then, the spectral model simulation of the GW amplitudes was applied to the 3-D field of SIMONe 2018 at a given time $t_{i}$ and position $\overrightarrow{r_{i}}$. In the following equation of the wind velocity, original SIMONe 2018 meteor positions and their time of occurrence were used to generate new velocities at these points in space and time.

$$
u_{\mathrm{sim}}=\sum_{i}^{n_{m}} \sum_{i}^{n_{\omega}} \overrightarrow{u_{i j}}(m, \omega) \sin \left(k_{i j} x+l_{i j} y+m_{i j} z-\omega_{i j} t+\phi_{i j}\right)
$$

where $u_{\operatorname{sim}}=\left(u_{\operatorname{sim}}, v_{\text {sim }}, w_{\text {sim }}\right)$ is the velocity vector of a point in space $(x, y, z, t)$ inside the simulation, $\overrightarrow{u_{i j}}$ is the amplitude of each component of the velocity obtained from the above mentioned GW spectral model simulation amplitude $(A(m, \omega)), k_{i j}, l_{i j}, m_{i j}$ are the wavenumbers in $\mathrm{x}, \mathrm{y}$ and $\mathrm{z}$ direction, $\omega_{i j}$ is the angular frequency of the wave and, $\phi_{i j}$ is the phase offset. Note that $(x, y, z)$ has been calculated from the meteor location information. The horizontal velocity amplitude $V_{h}$ was obtained by normalizing $A(m, \omega)$ using a normalizing factor, $c$. This factor was selected based on Spargo et al. (2019), where they used this normalization factor to get the mean values of momentum flux close to $20 \mathrm{~m}^{2} \mathrm{~s}^{-2}$, which are approximate values of $\langle u w\rangle$ and $\langle v w\rangle$ in the MLT region (Fritts et al. 2012).

$$
V_{h}=\sqrt{\left(\frac{A(m, \omega) c}{\sum A(m, \omega)}\right)}
$$

where $V_{h}=\sqrt{u^{2}+v^{2}}$. Considering the dispersion relation for GWs with medium intrinsic frequencies, we have,

$$
K_{h}=\left(\frac{m \omega}{N}\right)
$$

where $K_{h}=\sqrt{k^{2}+l^{2}}$ is the horizontal wavenumber, and $N$ is the Brunt-Väisälä frequency. The GW polarization relations under the medium frequency approximation was used to maintain the correlation between horizontal and vertical velocities (Fritts and Alexander 2003; Nappo 2012), i.e.,

$$
w=\left(\frac{V_{h} K_{h}}{m}\right)
$$

278 Figure 4a shows the dependence of the horizontal wavelength on period and vertical wavelength of the simulated waves. 
The wave propagation angle $\theta$ was used to determine the horizontal components of wavenumbers and velocities.

$$
\begin{gathered}
u=V_{h} \sin (\theta) ; v=V_{h} \cos (\theta) \\
k=K_{h} \sin (\theta) ; l=K_{h} \cos (\theta)
\end{gathered}
$$

Equation 5 was applied to the SIMONe 2018 meteor data positions and times of occurrence to obtain the three components of the velocity. The wave propagation azimuth $\theta$ and, phase offset $\phi$ were sampled from uniform random distributions in ranges of $[0, \pi]$ and $[0,2 \pi] \mathrm{rad}$ respectively. In the following step, the obtained $\overrightarrow{u_{\text {sim }}}$ was applied to get the new simulated Doppler velocities $\left(V_{r}^{\prime}\right)$ using the Bragg vector from the SIMONe 2018 data.

$$
V_{r}^{\prime}=u_{\operatorname{sim}}^{\vec{k}} \cdot \vec{k}
$$

The estimated velocities $\overrightarrow{u_{\text {est }}}=\left(u_{\text {est }}, v_{\text {est }}, w_{\text {est }}\right)$ were obtained after fitting the new simulated Doppler velocities $V_{r}^{\prime}$ at all meteor detections at a given altitude gate and a time bin using the existing Bragg vector $\vec{k}$ of SIMONe 2018 as described in methodology section (MWE). i.e., $\vec{u}_{\mathrm{est}}=\left(\vec{k}^{T} \vec{k}\right)^{-1} \vec{k}^{T} V_{r}^{\prime}$.

The major outcomes of the GW simulation are the new simulated Doppler velocity $V_{r}^{\prime}$ and the new estimated velocity $\overrightarrow{u_{\text {est }}}$. These parameters are then utilized to obtain frequency spectra using the WCFI and MWE methods which are examined below in the Results and Discussion sections.

\section{Results}

In this section, we introduce the results obtained from the Monte Carlo GW simulations and SIMONe 2018 observations using the WCFI and MWE methods. We start with spectral results from the simulations that were utilized to compare and validate both methods. Then we present the results obtained from SIMONe 2018 and compare the results obtained by the two methods, i.e., MWE and WCFI, followed by presenting the horizontal correlation results from SIMONe 2018.

\section{Spectral characteristics of the simulation}

Frequency spectra of the simulated winds were estimated using the MWE and WCFI methods. The orange dotted lines in Figure $4 \mathrm{~b}$ and $4 \mathrm{c}$ show the spectra used as an input in the simulation, as they results from equation 4 . In the simulation is assumed that the frequency spectrum has a slope of -2 . In the MWE method, the spectra were obtained by applying Fourier transform on the velocity $\overrightarrow{u_{\text {est }}}$ at each altitude. The blue dashed lines in Figures $4 \mathrm{~b}$ and $4 \mathrm{c}$ show the frequency spectra of horizontal wind obtained after averaging such individual spectra between 80 and $100 \mathrm{~km}$ altitude. Here, the spectra from 
the MWE method provided a steeper slope than the reference spectra.

In the WCFI method, the frequency spectra were estimated using the simulated Doppler velocity $V_{r}^{\prime}$ (see equation 10). We selected a 30-minute temporal lag and two different horizontal lag configurations for this analysis: $50 \mathrm{~km}$ and $500 \mathrm{~km}$. These selection criteria bounded the measurement pairs to have a maximum spatial separation $\Delta s=50 \mathrm{~km}$ or $\Delta s=500 \mathrm{~km}$ depending on the configuration. In both cases, the temporal correlations were averaged between 80 and $100 \mathrm{~km}$ in altitude. In the case of $\Delta s=50 \mathrm{~km}$, we expected it to provide a better estimation of the spectra due to their smaller horizontal separation compared to the sizeable averaging area used in the MWE spectral estimation. However, in the case of $\Delta s=500 \mathrm{~km}$, we expected it to give similar slopes to that of the MWE spectra due to comparable area averaging as in the MWE estimation. The solid black lines in Figures $4 \mathrm{~b}$ and $4 \mathrm{c}$ show the spectra estimated using these two configurations, $\Delta s=50 \mathrm{~km}$ and $\Delta s=500 \mathrm{~km}$ respectively. The spectra obtained by the WCFI method outperformed the MWE method in reproducing the reference spectra, as shown in Figure 4b. The spectra from the WCFI follows the reference spectrum with a -2 slope until around ( $2 \pi / 1.5$ hours). In the $500 \mathrm{~km}$ case, as shown in Figure 4c, the WCFI spectra provided a spectrum similar to that of the MWE method, as expected.

\section{Spectral characteristics of the SIMONe 2018 campaign data}

Here we present the spectral analysis of SIMONe 2018 using the WCFI method. In order to estimate the spectra, we allowed horizontal displacements of up to $50 \mathrm{~km}$ and temporal lag of $30 \mathrm{~min}$ between the meteor pairs within the total radar illuminated area. If one assumes the waves statistics to be the same over a relatively large area, then the 50-km lag can be obtained from different configurations, for example: (i) 0-50 km (ii) 50-100 km, and (iii) 100-150 km. In other words, in (i) 0-50 km, (ii) 50-100 km, and (iii) 100-150 km horizontal scale cases, we selected measurement pairs to have a spatial separation between 0 and $50 \mathrm{~km}, 50$ and $100 \mathrm{~km}$, and 100 and $150 \mathrm{~km}$, respectively. Figure 5 shows a two-dimensional histogram of horizontal and temporal lags of measurement pairs found between 92 and $93 \mathrm{~km}$ altitude. The black dashed line in the left side histogram of the temporal displacements in Figure 5 demonstrates the highest number of measurement pairs in the $100-150 \mathrm{~km}$ horizontal scale case.

Even though the number of meteor pairs is smaller in the 0-50 km horizontal scale case compared to the other two cases, WCFI was applied to determine temporal autocorrelation function since the number of pairs was large enough to ensure the convergence of the statistics. In all three cases, the analysis implemented average waves with horizontal wavelengths smaller than $50 \mathrm{~km}$ and presented the correlation 
of the waves with horizontal wavelength larger than $50 \mathrm{~km}$. If all the waves with horizontal wavelengths larger than $50 \mathrm{~km}$ are present in the MLT for more than $150 \mathrm{~km}$ in the horizontal, we expect the spectra to behave identically in these three cases. However, if some of these waves did not sustain for $150 \mathrm{~km}$ in space, we expect the three spectra to produce different slopes.

The three plots in Figure 6 show the horizontal frequency spectra with horizontal scales 0-50 km, 50$100 \mathrm{~km}$ and $100-150 \mathrm{~km}$, each of them estimated after averaging around $90( \pm 3) \mathrm{km}$ in altitude. In the plots, the solid orange lines correspond to the frequency spectra of horizontal velocity and the light green vertical lines in the spectra show the standard error from spectra estimation. The solid black lines show the power-law curve fitted to the spectra of horizontal winds between ( $2 \pi / 7$ hours) and ( $2 \pi /$ 2 hours) and their slope values are inscribed next to the slope line. The range within these slopes has more extension to the higher frequencies in the case of 100-150 km than in 0-50 km, which is due to the number of measurement pairs used. The slopes $(\approx-2.5)$ from three different horizontal analyses are similar, considering the standard error of curve fit estimation between the selected frequency range. Due to the high number of measurement pairs, and to ease the discussion henceforth, the paper will be focusing on the analysis only on 100-150 km horizontal scales.

\section{MWE vs WCFI horizontal wind frequency spectra}

The mean zonal and meridional winds from the MWE are shown in Figure 2. Mean zonal and mean meridional winds are dominated by semidiurnal tides (12 hours), as seen in Figure 2. The estimated velocities were used to obtain the frequency spectra around three different altitudes from the SIMONe 2018 winds, i.e., $84( \pm 3), 90( \pm 3), 96( \pm 3) \mathrm{km}$. As described in the methodology section, the Fourier transform was used on each horizontal component to obtain the spectra of the horizontal wind. The blue dashed lines in Figure 7 depict the spectra obtained using the MWE method. Figures 7a, 7b, 7c show the spectra of the horizontal winds around the three different altitudes. The solid blue lines above the horizontal spectra are of the power-law fits between $(2 \pi / 7 \mathrm{~h})$ and $(2 \pi / 2 \mathrm{~h})$ and, transparent blue shades on the solid lines depict the uncertainty of the power-law fit. The slope of the frequency spectra from the MWE horizontal winds are written on the lower left side of each plot in a blue colour font and appear to be increasing as altitude increases.

The solid black lines in Figure 7 depict the spectra in the frequency range obtained by the WCFI method around the same three altitude averages. These spectra were estimated with a 30-minute temporal lag and a spatial lag of 100 to $150 \mathrm{~km}(\Delta s=50 \mathrm{~km})$ between meteor observations. The results of the 
WCFI method are presented in a similar manner than the ones obtained by the MWE method but in black instead of blue. A power-law slope line of $-5 / 3$ (Kolmogorov 1941) is also depicted in those plots as a reference line. The spectral slopes are comparable at different altitudes within the standard error of the power-law fit. Horizontal frequency spectra around $84 \mathrm{~km}$ average gave a $-2.36 \pm 0.3$ slope, and average around $90 \mathrm{~km}$ altitude gave $-2.59 \pm 0.22$ slope, and $96 \mathrm{~km}$ average gave a $-2.56 \pm 0.29$ slope. The comparison of the spectra using SIMONe 2018 data obtained by the MWE and WCFI methods shows similar power-law slopes around different altitudes within the uncertainties of slope values. However, the comparative study of these two methods using the GW spectral model simulation showed a considerable difference between two slopes.

As part of the WCFI, the temporal correlation function of the vertical velocities is also obtained and therefore its corresponding frequency spectra. The main spectral amplitude for the vertical velocity peaks at a frequency corresponding to 24 hours, in agreement with the 24 hour pattern observed in the apparent vertical velocities obtained with the MWE method in Figure 2. Recall that the WCFI does not assume any functional form of the wind fields in space or time. As mentioned above, we are leaving the understanding of the obtained vertical velocities values and spectral features to a future work.

\section{Horizontal correlation functions of horizontal wind}

The spatial correlation functions of the zonal and meridional winds calculated using the WCFI based on the entire campaign period are shown in Figure 8. The analysis used the meteor pairs with a horizontallag of $20 \mathrm{~km}$ in a spatial domain of $400 \mathrm{~km}$ in the horizontal, $1 \mathrm{~km}$ in the vertical, and 1-hour temporal resolution. In each plot in Figure 8, the solid orange lines illustrate the horizontal autocorrelation function (ACF) of the four-hour high pass filtered components of the zonal (a, c, and e) and meridional winds (b, d, and f) during the whole seven days of the campaign. The analysis was carried out by averaging altitudes around $84( \pm 3), 90( \pm 3)$, and $96( \pm 3) \mathrm{km}$. The plots of ACF of seven days show a gradual decorrelation of the signals with the spatial separation. The solid purple line in the plots shows the ACF of the four-hour high pass filtered components of zonal and meridional wind components in a 12 hour period during which $\approx 3$-hour GWs were qualitatively identified (see Figure $2 \mathrm{~b}$ ) on the 7th of November 2018. This ACFs decays more slowly, with the signal never decorrelating at high-altitudes (Fig. 8). Error bars on each ACFs illustrate the standard error obtained from the analysis. Orange (7 days) and purple (12 hours) vertical lines and corresponding numbers correspond to the e-folding length scale $(1 / \alpha)$ with their uncertainties estimated by fitting the curves with an exponential function $f=a e^{-\alpha x}$, where $\mathrm{x}$ is 
the spatial lag. In Figure 8a, for the horizontal ACF of the zonal wind around $96( \pm 3) \mathrm{km}$ does not show the vertical line since the e-folding length is $520 \pm 25 \mathrm{~km}$, which is longer than $400 \mathrm{~km}$.

\section{Discussion}

The primary goals of this paper were to investigate the horizontal wind frequency spectra on different horizontal scales and their dependence with the MLT altitude during a seven-day multi-network meteor radar campaign. In this paper, the units that we have used for the spectra in the frequency domain correspond to those of the power spectral density. However, these spectra can be viewed as a function of kinetic energy spectra with the factor multiplication of the neutral density $(\rho)$, where $\rho$ is a function of altitude. The total energy of the waves consists of potential and kinetic energy. Previous studies of Arctic middle atmospheric winds and temperatures using Lidar measurements suggest that the kinetic energy at mesospheric heights is 4-5 times greater than the potential energy (Baumgarten et al. 2015; Hildebrand et al. 2017).

We have used the MWE and WCFI methods to estimate the frequency spectra, and they were implemented on a GW spectral model-simulated data, and the SIMONe 2018 campaign data. The analysis of the simulated data suggests that the WCFI method is more effective in reproducing the input spectra in comparison to the MWE method (Figure 4b,c). The usage of the measurement pairs instead of measurements by the WCFI method, allowed us to select smaller horizontal resolution while estimating the spectra. Hence in the simulation analyses, the WCFI with $\Delta s=50 \mathrm{~km}$ provided a better estimate of the spectra. Here, the smaller horizontal resolution $(50 \mathrm{~km})$ helped us to capture the energy of GWs with horizontal wavelengths larger than $50 \mathrm{~km}$. However, the MWE in the simulation provided a much steeper slope, and this is due to the large horizontal average (about $250 \mathrm{~km}$ radius) of meteors inherent to the MWE technique. This inference is evident when we compared the MWE spectra to the WCFI spectra with $500 \mathrm{~km}$ horizontal resolution, as seen in Figure 4c, where both the spectra provided similar slopes. Spectra obtained by WCFI $(\Delta s=50 \mathrm{~km})$ successfully reproduced the input spectra in the range from $(2 \pi / 8 \mathrm{~h})$ and $(2 \pi / 1.5 \mathrm{~h})$. Beyond $(2 \pi / 1.5 \mathrm{~h})$, the WCFI spectra reproduced a steeper slope than the input spectral slope (Figure 4b). An argument has been made that this decline in slopes after ( $2 \pi / 1.5 \mathrm{~h})$ is due to the horizontal scale $(50 \mathrm{~km})$ and temporal resolution (30 minutes) used in the WCFI analysis, which prevented the method to capture the energy of the small scale waves $(<50 \mathrm{~km}$ horizontal wavelength). However, the simulation results highlighted the high potential of the WCFI method to 
452 in further detail in Vargas et al. (2020). in further detail in Vargas et al. (2020).

estimate frequency spectra from multistatic meteor radar observations with respect to the MWE method. Based on our simulations, it is found that the WCFI method reproduces the energy of waves with horizontal scales smaller than the multistatic SMR observing volume, which is averaged out by the MWE method. This analysis confirms our speculations about the WCFI method, with which the energy of small scale GWs is better recorded than with the MWE spectra.

The comparison of the WCFI and MWE spectral analysis (Figure 7) based on the SIMONe 2018 data gave an unforeseen outcome comparing to the simulation result (Figure 4). A frequency range between $(2 \pi / 7 \mathrm{~h})$ to $(2 \pi / 2 \mathrm{~h})$ was selected for a power-law fit for altitude around $90( \pm 3)$ and $96( \pm 3) \mathrm{km}$. In the case of $84( \pm 3) \mathrm{km}$, a range between $(2 \pi / 7 \mathrm{~h})$ and $(2 \pi / 2.5 \mathrm{~h})$ in frequency has been selected due to the noise level after ( $2 \pi / 2.5 \mathrm{~h})$; however, for the comparative purpose, range of power-law slope was extrapolated until ( $2 \pi / 2 \mathrm{~h}$ ). In the spectra obtained by WCFI and MWE, the GW spectral slope was found to be statistically similar within the range of uncertainties around each altitude, which was somewhat unexpected. In the simulation, the MWE spectra exhibited a steeper slope than WCFI due to MWE method's limitations in capturing the energy of the small scale waves. We believe that the reason for this discrepancy with the campaign data is due to the dominance of large-scale GWs during the campaign period. It should also be noted that the four-hour high pass filtered mean zonal and mean meridional winds in Figure 2 shows the presence of highly dynamic GWs. These mean winds were obtained after averaging over the entire illuminated radar area with a radius of approximately $250 \mathrm{~km}$ using the MWE method. This implies that all wave-like structures in the mean wind plots of Figure 2 have horizontal wavelengths of more than $500 \mathrm{~km}$. It is also shown that wave-like structures in Figure $2 \mathrm{~b}$ and $2 \mathrm{~d}$ indicate the presence of waves with long vertical wavelengths $(>20 \mathrm{~km})$.

The horizontal autocorrelation analysis shows long correlation length at 12-hour analysis (Figure 8) around $96 \mathrm{~km}$ in altitude. This selected period for the analysis is illustrated by a black line box in Figure $2 \mathrm{~b}$, which demonstrate 3-4 hour GW signatures. Results from the spatial correlation analysis suggest that the GWs during the 12 hours have more than $400 \mathrm{~km}$ horizontal wavelength. Indeed, if we use the observed vertical wavelengths (more than $30 \mathrm{~km}$ ) and periods (close to 3 hours) of Figure 2, using the GW dispersion relation, the expected horizontal wavelength is larger than $1200 \mathrm{~km}$, which is discussed

Vargas et al. (2020) also shows the presence of large horizontal scale GWs by studying the airglow observations during the SIMONe 2018 campaign. They were also able to see a significant amount of 
small horizontal scale features with periods of less than 1 hour, which are out of the scope of our paper. Our study focuses on the MLT fluctuations with periods between 7 hours and 2 hours. Combining the results from the simulation and campaign data, it is clear that the large horizontal GWs with periods between 7 and 2 hours are dominant during the campaign. Recent studies of secondary GWs suggest that they have much larger horizontal wavelength compared to the primary GWs and mountain waves generated in the troposphere (Fritts et al. 2006; Vadas and Becker 2018; Vadas et al. 2018; Becker and Vadas 2018; Heale et al. 2020). Lidar and Radar observational studies from the winter Antarctic region also showed the presence of 3-10 hour dominant GWs with horizontal wavelengths of several thousands of kilometres in the MLT (Chen et al. 2013, 2016; Chen and Chu 2017). Later in a numerical modelling study of the secondary GWs, Vadas and Becker (2018) associated these dominant large scale waves presented during the wintertime at McMurdo Station in the Antarctic as secondary GWs in nature. Although our campaign was conducted in the mid-latitudes of the northern hemisphere pre-wintertime, we speculate that the large scale GWs dominating during our campaign period are probably due to the secondary GWs. However, due to the scales of observed waves in our study, we attribute them to secondary waves like those explained in Vadas and Becker (2018). We are currently collaborating on finding specific connections to secondary waves, like fish-bone structures in temperature fluctuations in the stratosphere, and the possible sources of the primary waves causing them.

Our work motivates future studies of the secondary GWs in the MLT region using various observational techniques and also look for a month to month variability study of the wind spectra by analyzing longterm observational data in the MLT. The WCFI method can be used to study the spatial spectra provided that we have enough meteor radar measurement coverage in the horizontal dimension. In the current study, we have conducted only the frequency spectral analysis of the horizontal winds due to the limited number of days of the campaign. In the future, we will investigate the seasonal variability of Reynolds stress tensor terms (power spectra and momentum fluxes) by analyzing a multiyear multistatic SMR data from the MMARIA-Germany system since September 2018.

Although vertical velocities are not the main trust of the paper, here we briefly discuss our estimated values. The apparent vertical winds estimated using the gradient method present relatively large amplitudes lasting for a few hours (Figure 2). Chau et al. (2017) communicated that the monostatic meteor radars should not be used to estimate vertical winds since they are contaminated by horizontal divergence. However, the frequency spectra of apparent vertical winds obtained with the WCFI during the 
campaign period demonstrated similar amplitudes with a dominant 24-hour period, where the horizontal components showed a dominant peak at 12 hours. Diurnal structures in the vertical velocity have also been observed in other multistatic meteor radar systems in Peru and Argentina (Chau et al. 2021; Conte et al. 2021). Vertical velocities with magnitudes of between $5-15 \mathrm{~ms}^{-1}$ lasting for a few hours have been observed with other instruments around $100 \mathrm{~km}$ altitude (Larsen and Meriwether 2012). Specular meteor radars were also previously shown to have higher amplitudes in the vertical velocity estimates after averaging a narrow region (e.g., Babu et al. 2012; Egito et al. 2016). In our study, we are observing high amplitudes after considering a horizontal area with $250 \mathrm{~km}$ radius. We speculate one reason for vertical estimates with high temporal and altitude variability might be the horizontal variability of the horizontal wind even after applying the gradient approach. However, this does not explain the diurnal structure in the estimates. We are currently working on validating the vertical estimates of our multistatic radar analysis on high-resolution atmospheric models to investigate the reasons of our unexpected vertical estimates. Until then, we are not claiming these vertical velocities as real atmospheric motions; hence, the estimates of vertical velocities are referred to as "apparent vertical winds".

\section{Conclusions}

In this work, we have presented the frequency spectra of horizontal MLT winds from a seven-day multistatic meteor radar campaign called SIMONe 2018. The campaign comprised of fourteen meteor radar links located in the northern part of Germany, which resulted in more than a hundred thousand specular meteor detections per day.

The study focused on wind field correlation function and mean wind estimation methods on estimating the horizontal wind frequency spectra. The validation analysis based on Monte Carlo simulations of a GW spectral model suggested that the WCFI method outperformed the MWE method in reproducing the frequency spectra of the horizontal wind. The WCFI method allowed us to choose a horizontal scale of $50 \mathrm{~km}$ to estimate the spectra; hence the method was able to estimate the energy of small scale GWs with horizontal wavelengths larger than $50 \mathrm{~km}$ and periods greater than 1 hour. The MWE method inherently uses the total radar illuminated area to estimate the mean winds; hence the method was only able to acquire the energy of horizontal structures much larger than $500 \mathrm{~km}$.

The used WCFI and MWE analysis on SIMONe 2018 data exhibited an unexpected result. In the simulation, the frequency spectra demonstrated a significant difference in power-law slopes between the 
spectra estimated by WCFI and MWE methods, but the campaign data analysis gave similar spectral slopes at each altitude range when using the two methods. The horizontal autocorrelation analysis of SIMONe 2018 data during a 12 hour time interval of 3-hour GW presence, suggested that these waves have long horizontal wavelengths. Based on the results from the simulation and campaign observations, we speculate that the MLT region during the period of the campaign was dominated by large scale waves of horizontal wavelengths much larger than $500 \mathrm{~km}$, which probably are due to secondary GWs.

\section{Declarations}

\section{Availability of data and materials}

The data used to generate the figures presented in this paper can be found in HDF5 format at DOI: $10.22000 / 456$.

\section{Competing interests \\ The authors declare that they have no competing interests.}

\section{Funding \\ This work was partially supported by the Deutsche Forschungsgemeinschaft (DFG, German Research Foundation) under SPP 1788 (DynamicEarth)-CH1482/2-1 and under SPP 1788 (CoSIP)-CH1482/3- 1, by the WATILA Project (SAW-2015-IAP-1) and MSGWaves/PACOG project LU 1174/8-1. Some hardware, software, and analysis work at MIT Haystack Observatory was supported by NSF Major Research Infrastructure Grant AGS-1626041. HCA appreciate the support by the French Ministry of Foreign and European Affairs for the Eiffel excellence scholarship (File N 945179K). RM acknowledges support from the project "EVENTFUL" (ANR-20-CE30-0011), funded by the French "Agence Nationale de la Recherche" - ANR through the program AAPG-2020.}

\section{Authors' contributions}

HCA implemented the simulations, analyzed the data, wrote most of the paper. JLC conceived the idea of the campaign and help with the interpretation of the results. RM contribute to the interpretation of the results with particular emphasis to waves and turbulence. JV wrote the main routines related to the WCFI method and help with the interpretation and analysis of the spatial correlation functions. JMU 


\section{Acknowledgments}

We thank Rüdiger Lange (Salzwedel), Fred and Claudia Bauske (Mechelsdorf), Frank Schütz (Gulderup), Dieter Keuer (Breege), and the IAP personnel T. Barth, F. Conte, N. Gudadze, R. Latteck, N. Pfeffer, and J. Trautner for supporting the operations of the coded CW links.

\section{References}

Alexander, M. J., Geller, M., McLandress, C., Polavarapu, S., Preusse, P., Sassi, F., Sato, K., Eckermann, S., Ern, M., Hertzog, A., Kawatani, Y., Pulido, M., Shaw, T. A., Sigmond, M., Vincent, R., and Watanabe, S. (2010). Recent developments in gravity-wave effects in climatemodels and the global distribution of gravity-wavemomentum flux from observations and models.

Alexander, S. P., Klekociuk, A. R., and Murphy, D. J. (2011). Rayleigh lidar observations of gravity wave activity in the winter upper stratosphere and lower mesosphere above Davis, Antarctica $\left(69^{\circ} \mathrm{S}\right.$, $\left.78^{\circ} \mathrm{E}\right)$. Journal of Geophysical Research, 116(D13):D13109.

Antonita, T. M., Ramkumar, G., Kumar, K. K., and Deepa, V. (2008). Meteor wind radar observations of gravity wave momentum fluxes and their forcing toward the Mesospheric Semiannual Oscillation. Journal of Geophysical Research, 113(D10):D10115.

Babu, V. S., Ramkumar, G., and John, S. R. (2012). Seasonal variation of planetary wave momentum flux and the forcing towards mean flow acceleration in the MLT region. Journal of Atmospheric and Solar-Terrestrial Physics, 78-79(C):53-61.

Balsley, B. B. and Garello, R. (1985). The kinetic energy density in the troposphere, stratosphere and mesosphere: A preliminary study using the Poker Flat MST radar in Alaska. Radio Science, 20(6):1355-1361.

Baumgarten, G. (2010). Atmospheric Measurement Techniques Doppler Rayleigh/Mie/Raman lidar for wind and temperature measurements in the middle atmosphere up to $80 \mathrm{~km}$. Atmos. Meas. Tech, 3:1509-1518.

Baumgarten, G., Fiedler, J., Hildebrand, J., and Lübken, F.-J. (2015). Inertia gravity wave in the 
stratosphere and mesosphere observed by Doppler wind and temperature lidar. Geophysical Research Letters, 42(24):929-10.

Becker, E. and Vadas, S. L. (2018). Secondary Gravity Waves in the Winter Mesosphere: Results From a High-Resolution Global Circulation Model. Journal of Geophysical Research: Atmospheres, $123(5): 2605-2627$.

Browning, K. A. and Wexler, R. (1968). The determination of kinematic properties of a wind field using \{Doppler\} radar. J. Appl. Meteorol., 7(1):105-113.

Burnside, R. G., Herrero, F. A., Meriwether, J. W., and Walker, J. C. G. (1981). Optical observations of thermospheric dynamics at Arecibo. Journal of Geophysical Research, 86:5532-5540.

Chanin, M.-L. and Hauchecorne, A. (1981). Lidar observation of gravity and tidal waves in the stratosphere and mesosphere. Journal of Geophysical Research, 86(C10):9715.

Chau, J. L. and Clahsen, M. (2019). Empirical Phase Calibration for Multistatic Specular Meteor Radars Using a Beamforming Approach. Radio Science, 54(1):60-71.

Chau, J. L., Stober, G., Hall, C. M., Tsutsumi, M., Laskar, F. I., and Hoffmann, P. (2017). Polar mesospheric horizontal divergence and relative vorticity measurements using multiple specular meteor radars. Radio Science, 52(7):811-828.

Chau, J. L., Urco, J. M., Vierinen, J., Harding, B. J., Clahsen, M., Pfeffer, N., Kuyeng, K. M., Milla, M. A., and Erickson, P. J. (2021). Multistatic Specular Meteor Radar Network in Peru: System Description and Initial Results. Earth and Space Science, 8(1):e2020EA001293.

Chau, J. L., Urco, J. M., Vierinen, J. P., Volz, R. A., Clahsen, M., Pfeffer, N., and Trautner, J. (2019). Novel specular meteor radar systems using coherent MIMO techniques to study the mesosphere and lower thermosphere. Atmospheric Measurement Techniques, 12:2113-2127.

Chen, C. and Chu, X. (2017). Two-dimensional Morlet wavelet transform and its application to wave recognition methodology of automatically extracting two-dimensional wave packets from lidar observations in Antarctica. Journal of Atmospheric and Solar-Terrestrial Physics, 162:28-47.

Chen, C., Chu, X., McDonald, A. J., Vadas, S. L., Yu, Z., Fong, W., and Lu, X. (2013). Inertia-gravity waves in Antarctica: A case study using simultaneous lidar and radar measurements at McMurdo/Scott Base $\left(77.8^{\circ} \mathrm{S}, 166.7^{\circ} \mathrm{E}\right)$. Journal of Geophysical Research: Atmospheres, 118(7):2794-2808.

Chen, C., Chu, X., Zhao, J., Roberts, B. R., Yu, Z., Fong, W., Lu, X., and Smith, J. A. (2016). Lidar 
observations of persistent gravity waves with periods of 3-10 $\mathrm{h}$ in the Antarctic middle and upper atmosphere at McMurdo $\left(77.83^{\circ} \mathrm{S}, 166.67^{\circ} \mathrm{E}\right)$. Journal of Geophysical Research A: Space Physics, 121(2):1483-1502.

Conde, M. and Smith, R. W. (1998). Spatial structure in the thermospheric horizontal wind above \{Poker Flat, Alaska\}, during solar minimum. J. Geophys. Res., 103:9449-9472.

Conte, J. F., Chau, J. L., Urco, J. M., Latteck, R., Vierinen, J., and Salvador, J. O. (2021). First Studies of Mesosphere and Lower Thermosphere Dynamics Using a Multistatic Specular Meteor Radar Network Over Southern Patagonia. Earth and Space Science, 8(2):e2020EA001356.

Egito, F., Andrioli, V. F., and Batista, P. P. (2016). Vertical winds and momentum fluxes due to equatorial planetary scale waves using all-sky meteor radar over Brazilian region. Journal of Atmospheric and Solar-Terrestrial Physics, 149:108-119.

Espy, P. J., Jones, G. O. L., Swenson, G. R., Tang, J., and Taylor, M. J. (2004). Seasonal variations of the gravity wave momentum flux in the Antarctic mesosphere and lower thermosphere. Journal of Geophysical Research: Atmospheres, 109(D23):1-9.

Feraco, F., Marino, R., Primavera, L., Pumir, A., Mininni, P. D., Rosenberg, D., Pouquet, A., Foldes, A., Lévêque, E., Camporeale, E., Cerri, S., Charuvil Asokan, H., Chau, J. L., Bertoglio, J., Salizzoni, P., and Marro, M. (2021). Connecting large-scale velocity and temperature bursts with small-scale intermittency in stratified turbulence. EPL (Europhysics Letters).

Feraco, F., Marino, R., Pumir, A., Primavera, L., Mininni, P. D., Pouquet, A., and Rosenberg, D. (2018). Vertical drafts and mixing in stratified turbulence: Sharp transition with Froude number. EPL (Europhysics Letters), 123(4):44002.

Fritts, D. C. and Alexander, M. J. (2003). Gravity wave dynamics and effects in the middle atmosphere. Reviews of Geophysics, 41(1):1003.

Fritts, D. C., Janches, D., and Hocking, W. K. (2010). Southern Argentina Agile Meteor Radar: Initial assessment of gravity wave momentum fluxes. Journal of Geophysical Research, 115(D19):D19123.

Fritts, D. C., Janches, D., Hocking, W. K., Mitchell, N. J., and Taylor, M. J. (2012). Assessment of gravity wave momentum flux measurement capabilities by meteor radars having different transmitter power and antenna configurations. Journal of Geophysical Research: Atmospheres, 117(D10):n/a-n/a.

Fritts, D. C., Vadas, S. L., Wan, K., and Werne, J. A. (2006). Mean and variable forcing of the middle atmosphere by gravity waves. Journal of Atmospheric and Solar-Terrestrial Physics, 68(3-5):247-265. 
Gage, K. S. and Balsley, B. B. (1984). MST radar studies of wind and turbulence in the middle atmosphere. Journal of Atmospheric and Terrestrial Physics, 46(9):739-753.

Gardner, C. S., Hostetler, C. A., and Franke, S. J. (1993). Gravity wave models for the horizontal wave number spectra of atmospheric velocity and density fluctuations. Journal of Geophysical Research: Atmospheres, 98(D1):1035-1049.

Gardner, C. S. and Voelz, D. G. (1987). Lidar studies of the nighttime sodium layer over Urbana, Illinois: 2. Gravity waves. Journal of Geophysical Research, 92(A5):4673.

Geller, M. A., Alexander, J. J., Love, P. T., Bacmeister, J., Ern, M., Hertzog, A., Manzini, E., Preusse, P., Sato, K., Scaife, A. A., and Zhou, T. (2013). A comparison between gravity wave momentum fluxes in observations and climate models. Journal of Climate, 26(17):6383-6405.

Heale, C. J., Bossert, K., Vadas, S. L., Hoffmann, L., Dörnbrack, A., Stober, G., Snively, J. B., and Jacobi, C. (2020). Secondary Gravity Waves Generated by Breaking Mountain Waves Over Europe. Journal of Geophysical Research: Atmospheres, 125(5).

Herbert, C., Marino, R., Rosenberg, D., and Pouquet, A. (2016). Waves and vortices in the inverse cascade regime of stratified turbulence with or without rotation. Journal of Fluid Mechanics, 806:165-204.

Hildebrand, J., Baumgarten, G., Fiedler, J., and Lübken, F.-J. (2017). Winds and temperatures of the Arctic middle atmosphere during January measured by Doppler lidar. Atmospheric Chemistry and Physics, 17(21):13345-13359.

Hocking, W., Fuller, B., and Vandepeer, B. (2001). Real-time determination of meteor-related parameters utilizing modern digital technology. Journal of Atmospheric and Solar-Terrestrial Physics, 63(2-3):155-169.

Hocking, W. K. (2005). A new approach to momentum flux determinations using SKiYMET meteor radars. Annales Geophysicae, 23(7):2433-2439.

Holdsworth, D. A., Reid, I. M., and Cervera, M. A. (2004). Buckland Park all-sky interferometric meteor radar. Radio Science, 39(5):n/a-n/a.

Holton, J. R. (1983). The Influence of Gravity Wave Breaking on the General Circulation of the Middle Atmosphere. Journal of the Atmospheric Sciences, 40(10):2497-2507.

Jones, J., Webster, A. R., and Hocking, W. K. (1998). An improved interferometer design for use with meteor radars. Radio Science, 33(1):55-65. 
Kaifler, N., Kaifler, B., Ehard, B., Gisinger, S., Dörnbrack, A., Rapp, M., Kivi, R., Kozlovsky, A., Lester, M., and Liley, B. (2017). Observational indications of downward-propagating gravity waves in middle atmosphere lidar data. Journal of Atmospheric and Solar-Terrestrial Physics, 162:16-27.

Khintchine, A. (1934). Korrelationstheorie der stationären stochastischen Prozesse. Mathematische Annalen, 109(1):604-615.

Kim, Y.-J., Eckermann, S. D., and Chun, H. Y. (2003). An overview of the past, present and future of gravity-wave drag parametrization for numerical climate and weather prediction models. Atmosphere - Ocean, 41(1):65-98.

Kolmogorov, A. N. (1941). The local structure of turbulence in incompressible viscous fluid for very large reynolds numbers. Dokl. Akad. Nauk SSSR, 30:301-305.

Larsen, M. F., Fukao, S., Aruga, O., Yamanaka, M. D., Tsuda, T., and Kato, S. (1991). A comparison of VHF radar vertical-velocity measurements by a direct vertical-beam method and by a VAD technique. Journal of Atmospheric and Oceanic Technology, 8:766-776.

Larsen, M. F. and Meriwether, J. W. (2012). Vertical winds in the thermosphere. Journal of Geophysical Research, 117(A9).

Lindzen, R. S. (1981). Turbulence and stress owing to gravity wave and tidal breakdown. Journal of Geophysical Research: Oceans, 86(C10):9707-9714.

Liu, H. L. (2019). Quantifying gravity wave forcing using scale invariance. Nature Communications, $10(1): 1-12$.

Liu, H.-L., McInerney, J. M., Santos, S., Lauritzen, P. H., Taylor, M. A., and Pedatella, N. M. (2014). Gravity waves simulated by high-resolution Whole Atmosphere Community Climate Model. Geophysical Research Letters, 41(24):9106-9112.

Lu, X., Chu, X., Li, H., Chen, C., Smith, J. A., and Vadas, S. L. (2017). Statistical characterization of high-to-medium frequency mesoscale gravity waves by lidar-measured vertical winds and temperatures in the MLT. Journal of Atmospheric and Solar-Terrestrial Physics, 162:3-15.

Manning, L., Villard, O., and Peterson, A. (1950). Meteoric Echo Study of Upper Atmosphere Winds. Proceedings of the IRE, 38(8):877-883.

Manson, A. H., Meek, C. E., Hall, C., Hocking, W. K., Macdougall, J., Franke, S., Igarashi, K., Riggin, D., Fritts, D. C., and Vincent, R. A. (1999). Gravity wave spectra, directions and wave interactions: Global MLT-MFR network. Technical report. 
Marino, R., Mininni, P., Rosenberg, D., and Pouquet, A. (2013). Inverse cascades in rotating stratified turbulence: fast growth of large scales. Europhys. Lett., 102:44006.

Marino, R., Mininni, P. D., Rosenberg, D. L., and Pouquet, A. (2014). Large-scale anisotropy in stably stratified rotating flows. Phys. Rev. E, 90(2):23018.

Marino, R., Pouquet, A., and Rosenberg, D. (2015a). Resolving the paradox of oceanic large-scale balance and small-scale mixing. Physical Review Letters, 114(11).

Marino, R., Rosenberg, D., Herbert, C., and Pouquet, A. (2015b). Interplay of waves and eddies in rotating stratified turbulence and the link with kinetic-potential energy partition Related content. EPL (Europhysics Letters), 112(4):49001.

McLandress, C. (1998). On the importance of gravity waves in the middle atmosphere and their parameterization in general circulation models. Journal of Atmospheric and Solar-Terrestrial Physics, 60(14):1357-1383.

Medeiros, A. F., Taylor, M. J., Takahashi, H., Batista, P. P., and Gobbi, D. (2003). An investigation of gravity wave activity in the low-latitude upper mesosphere: Propagation direction and wind filtering. Journal of Geophysical Research D: Atmospheres, 108(14).

Meriwether, J., Faivre, M., Fesen, C., Sherwood, P., and Veliz, O. (2008). New results on equatorial thermospheric winds and the midnight temperature maximum. Ann. Geophys, 26:447-466.

Nakamura, T., Tsuda, T., Yamamoto, M., Fukao, S., and Kato, S. (1993). Characteristics of gravity waves in the mesosphere observed with the middle and upper atmosphere radar: 2. Propagation direction. Journal of Geophysical Research: Atmospheres, 98(D5):8911-8923.

Nappo, C. J. (2012). International Geophysics, Volume 102 : Introduction to Atmospheric Gravity Waves (2nd Edition). Academic Press, Saint Louis, MO, USA.

Placke, M., Hoffmann, P., Becker, E., Jacobi, C., Singer, W., and Rapp, M. (2011a). Gravity wave momentum fluxes in the MLT-Part II: Meteor radar investigations at high and midlatitudes in comparison with modeling studies. Journal of Atmospheric and Solar-Terrestrial Physics, 73(9):911-920.

Placke, M., Hoffmann, P., Latteck, R., and Rapp, M. (2015). Gravity wave momentum fluxes from MF and meteor radar measurements in the polar MLT region. Journal of Geophysical Research-Space Physics, 120:736-750.

Placke, M., Stober, G., and Jacobi, C. (2011b). Gravity wave momentum fluxes in the MLT-Part I: 
Seasonal variation at Collm $\left(51.3^{\circ} \mathrm{N}, 13.0^{\circ} \mathrm{E}\right)$. Journal of Atmospheric and Solar-Terrestrial Physics, 73(9):904-910.

Pouquet, A., Marino, R., Mininni, P. D., and Rosenberg, D. (2017). Dual constant-flux energy cascades to both large scales and small scales. Physics of Fluids, 29(11):111108.

Reid, I. M. (1990). Radar observtions of stratified layers in the mesosphere and lower thermosphere (50-100 km). Advances in Space Research, 10(10):7-19.

Reid, I. M., McIntosh, D. L., Murphy, D. J., and Vincent, R. A. (2018). Mesospheric radar wind comparisons at high and middle southern latitudes. Earth, Planets and Space, 70(1):84.

Rosenberg, D., Pouquet, A., Marino, R., and Mininni, P. D. (2015). Evidence for Bolgiano-Obukhov scaling in rotating stratified turbulence using high-resolution direct numerical simulations. Physics of Fluids, 27(5):55105.

Sato, K., Kohma, M., Tsutsumi, M., and Sato, T. (2017). Frequency spectra and vertical profiles of wind fluctuations in the summer Antarctic mesosphere revealed by MST radar observations. Journal of Geophysical Research, 122(1):3-19.

Senft, D. C. and Gardner, C. S. (1991). Seasonal variability of gravity wave activity and spectra in the mesopause region at Urbana. Journal of Geophysical Research, 96(D9):17229.

Spargo, A. J., Reid, I. M., and MacKinnon, A. D. (2019). Multistatic meteor radar observations of gravity-wave-tidal interaction over southern Australia. Atmospheric Measurement Techniques, 12(9):4791-4812.

Stober, G. and Chau, J. L. (2015). A multistatic and multifrequency novel approach for specular meteor radars to improve wind measurements in the MLT region. Radio Science, 50(5):431-442.

Strelnikova, I., Baumgarten, G., and Lübken, F.-J. (2020). Advanced hodograph-based analysis technique to derive gravity-wave parameters from lidar observations. Atmospheric Measurement Techniques, 13(2):479-499.

Swenson, G. R. and Mende, S. B. (1994). OH emission and gravity waves (including a breaking wave) in all-sky imagery from Bear Lake, UT. Geophysical Research Letters, 21(20):2239-2242.

Taylor, M. J., Pautet, P. D., Fritts, D. C., Kaifler, B., Smith, S. M., Zhao, Y., Criddle, N. R., McLaughlin, P., Pendleton, W. R., McCarthy, M. P., Hernandez, G., Eckermann, S. D., Doyle, J., Rapp, M., Liley, B., and Russell, J. M. (2019). Large-Amplitude Mountain Waves in the Mesosphere Observed on 21 June 2014 During DEEPWAVE: 1. Wave Development, Scales, Momentum Fluxes, 
and Environmental Sensitivity. Journal of Geophysical Research: Atmospheres, 124(19):10364-10384.

Thorsen, D., Franke, S. J., and Kudeki, E. (1997). A new approach to $\{\mathrm{MF}\}$ radar interferometry for estimating mean winds and momentum flux. Radio $\sim$ Sci., 32(2):707-726.

Tsutsumi, M., Sato, K., Sato, T., Kohma, M., Nakamura, T., Nishimura, K., and Tomikawa, Y. (2017). Characteristics of Mesosphere Echoes over Antarctica Obtained Using PANSY and MF Radars. SOLA, 13A(Special_Edition):19-23.

Urco, J. M., Chau, J. L., Milla, M. A., Vierinen, J. P., and Weber, T. (2018). Coherent MIMO to improve aperture synthesis radar imaging of field-aligned irregularities: First results at Jicamarca. IEEE Transactions on Geoscience and Remote Sensing, 56(5):2980-2990.

Urco, J. M., Chau, J. L., Weber, T., Vierinen, J., and Volz, R. (2019). Sparse signal recovery in MIMO specular meteorradars with waveform diversity. IEEE Trans. Geosci. Remote Sensing, page 1.

Vadas, S. L. and Becker, E. (2018). Numerical Modeling of the Excitation, Propagation, and Dissipation of Primary and Secondary Gravity Waves during Wintertime at McMurdo Station in the Antarctic. Journal of Geophysical Research: Atmospheres, 123(17):9326-9369.

Vadas, S. L., Zhao, J., Chu, X., and Becker, E. (2018). The Excitation of Secondary Gravity Waves From Local Body Forces: Theory and Observation. Journal of Geophysical Research: Atmospheres, 123(17):9296-9325.

Vargas, F. (2019). Uncertainties in gravity wave parameters, momentum fluxes, and flux divergences estimated from multi-layer measurements of mesospheric nightglow layers. Advances in Space Research, 63(2):967-985.

Vargas, F., Chau, J., Asokan, H. C., and Gerding, M. (2020). Mesospheric gravity wave activity estimated via airglow imagery, meteor radar, and saber data taken during the simone-2018 campaign. Atmospheric Chemistry and Physics, this issue.

Vargas, F., Swenson, G., Liu, A., and Gobbi, D. (2007). O(1S), OH, and O2(b) airglow layer perturbations due to AGWs and their implied effects on the atmosphere. Journal of Geophysical Research: Atmospheres, 112(D14).

Vargas, F., Swenson, G., Liu, A., and Pautet, D. (2016). Evidence of the excitation of a ring-like gravity wave in the mesosphere over the Andes Lidar Observatory. Journal of Geophysical Research: Atmospheres, 121(15):8896-8912.

Vierinen, J., Chau, J. L., Charuvil, H., Urco, J. M., Clahsen, M., Avsarkisov, V., Marino, R., and Volz, 
R. (2019). Observing Mesospheric Turbulence With Specular Meteor Radars: A Novel Method for Estimating Second-Order Statistics of Wind Velocity. Earth and Space Science, 6(7):1171-1195.

Vierinen, J., Chau, J. L., Pfeffer, N., Clahsen, M., and Stober, G. (2016). Coded continuous wave meteor radar. Atmospheric Measurement Techniques, 9(2):829-839.

Vincent, R. A. and Reid, I. M. (1983). HF Doppler Measurements of Mesospheric Gravity Wave Momentum Fluxes. Journal of the Atmospheric Sciences, 40(5):1321-1333.

Waldteufel, P. and Corbin, H. (1979). On the Analysis of Single-Doppler Radar Data. J. Appl. Meteorol., 18(2):532-542.

Weinstock, J. (1996). Spectra and a global source of gravity waves for the middle atmosphere. Advances in Space Research, 17(11):67-76.

Wiener, N. (1930). Generalized harmonic analysis. Acta Mathematica, 55(1):117-258.

Wüst, S., Offenwanger, T., Schmidt, C., Bittner, M., Jacobi, C., Stober, G., Yee, J.-H., Mlynczak, M. G., and Russell III, J. M. (2017). Derivation of horizontal and vertical wavelengths using a scanning $\mathrm{OH}(3-1)$ airglow spectrometer. Atmospheric Measurement Techniques Discussions, pages $1-26$.

Yasui, R., Sato, K., and Tsutsumi, M. (2016). Seasonal and Interannual Variation of Mesospheric Gravity Waves Based on MF Radar Observations over 15 Years at Syowa Station in the Antarctic. SOLA, 12(0):46-50.

Yiğit, E. and Medvedev, A. S. (2015). Internal wave coupling processes in Earth's atmosphere. Younger, J. P. and Reid, I. M. (2017). Interferometer angle-of-arrival determination using precalculated phases. Radio Science, 52(9):1058-1066.

Zhou, Q. H. (2000). Incoherent scatter radar measurement of vertical winds in the mesosphere. Geophysical Research Letters, 27(12):1803-1806. 

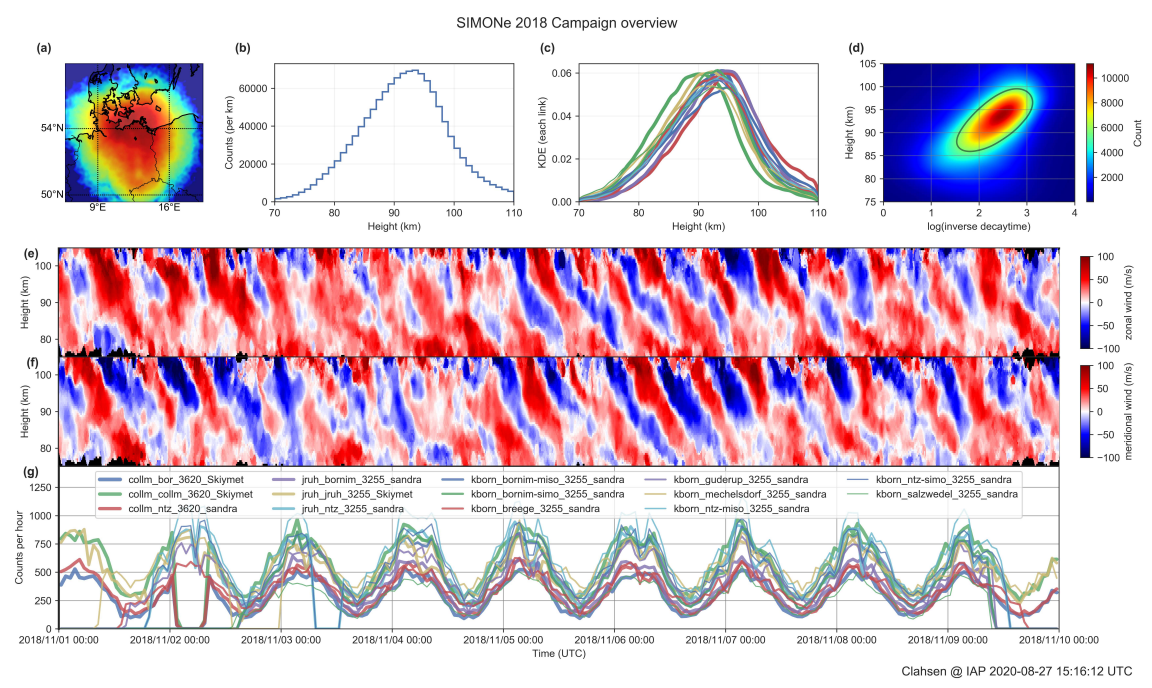

Figure 1. Example of parameters obtained after combining the fourteen SIMONe 2018 links: (a) 2D histogram of detections on latitude vs longitude axes, (b) altitude distributions across all links, (c) altitude distribution of each link, (d) 2D histogram altitude vs inverse decay time, (e) mean zonal winds, (f) mean meridional winds, and (g) counts per hour for each bistatic link. 
(a) Zonal mean wind
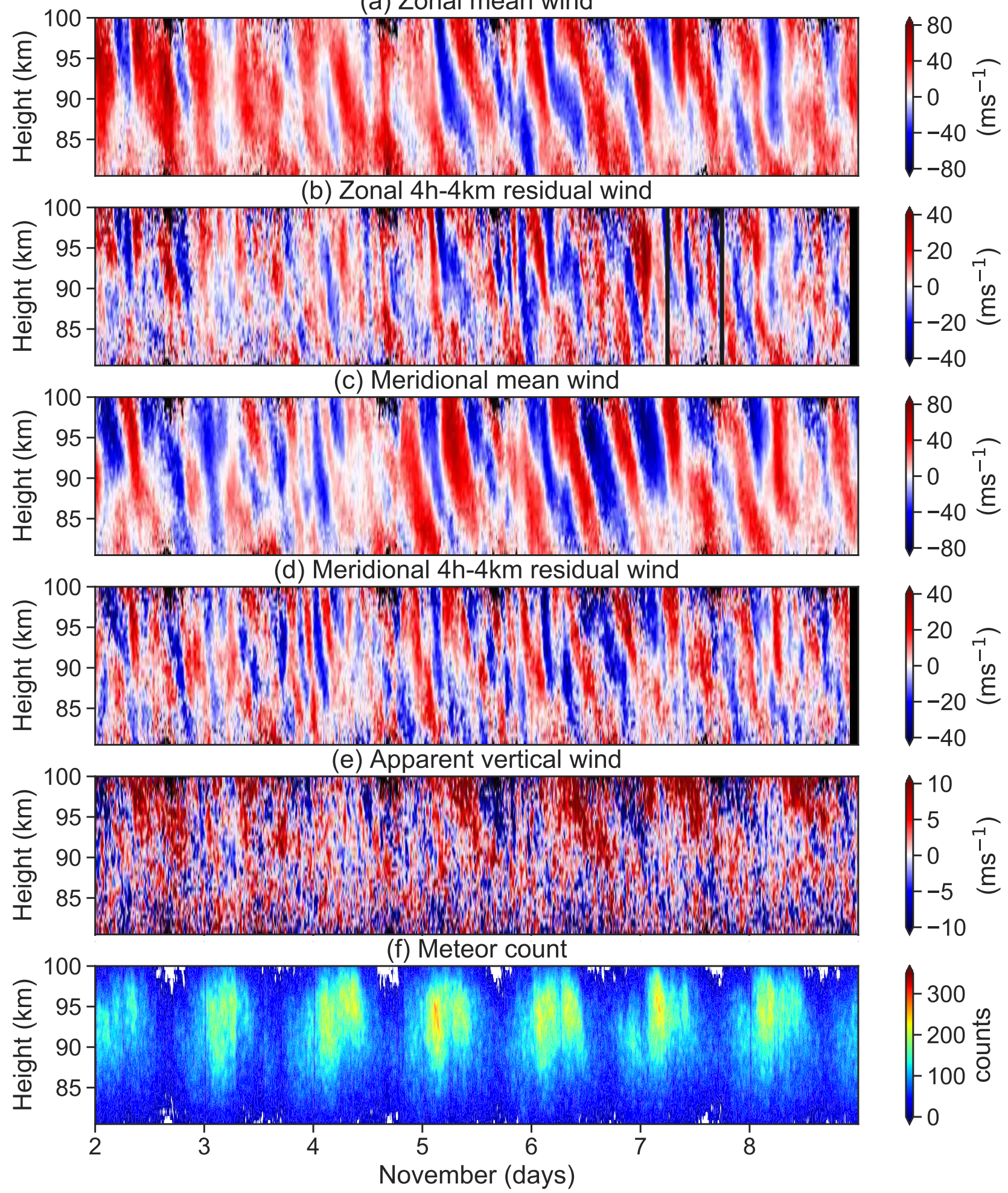

Figure 2. The figure 2 (a-e) shows the mean zonal, residual zonal, mean meridional, residual meridional, and apparent vertical wind velocities estimated from the SIMONe 2018 Campaign data from Nov 2 to Nov 9, 2018. Panel (f) shows the meteor counts during the campaign period. Mean winds were estimated using specular meteor events that occurred at a given altitude during 30 minutes and $1 \mathrm{~km}$ time-altitude bins and inside the total radar illuminated area. 

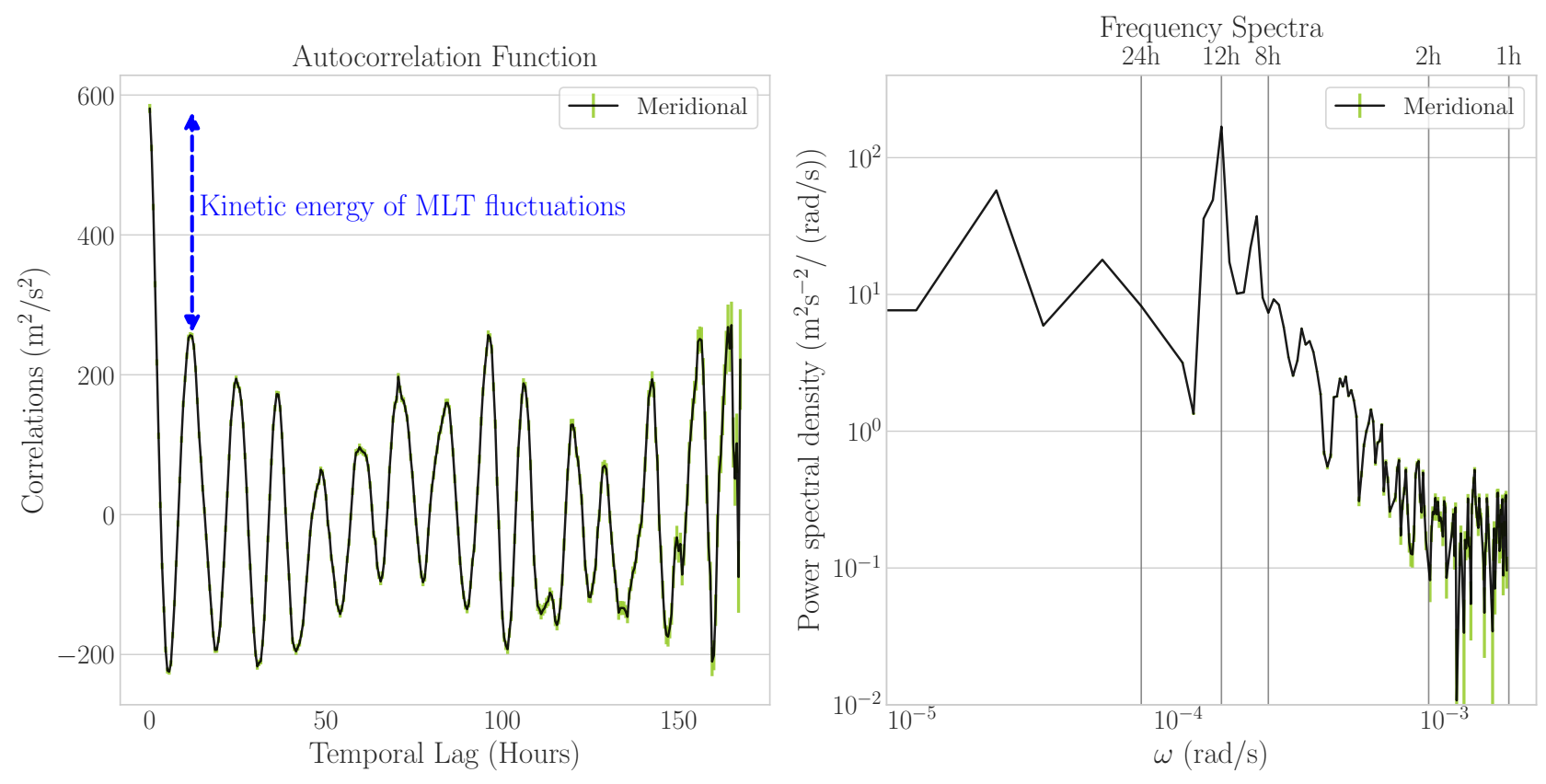

Figure 3. The Figure shows the autocorrelation function, and frequency spectra of the meridional wind component derived using the WCFI method. The autocorrelation function was estimated by averaging over a range of 87 to $93 \mathrm{~km}$ in altitude with 1800 seconds (30 min) temporal lag, $50 \mathrm{~km}$ horizontal lag and $1 \mathrm{~km}$ vertical lag resolutions 
(a)
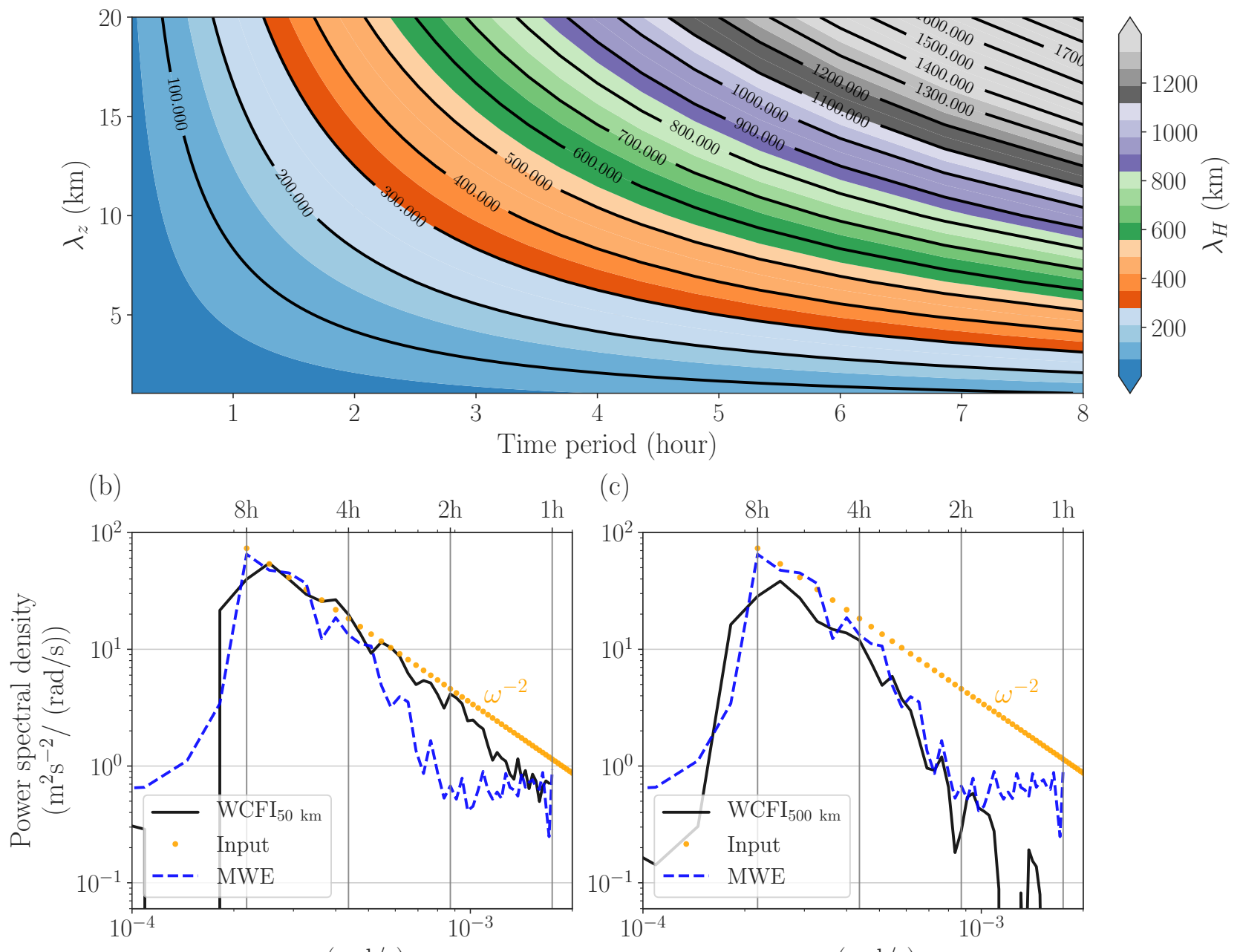

(c) $\quad 8 \mathrm{~h} \quad 4 \mathrm{~h} \quad 2 \mathrm{~h} \quad 1 \mathrm{~h}$

$$
\omega(\mathrm{rad} / \mathrm{s})
$$

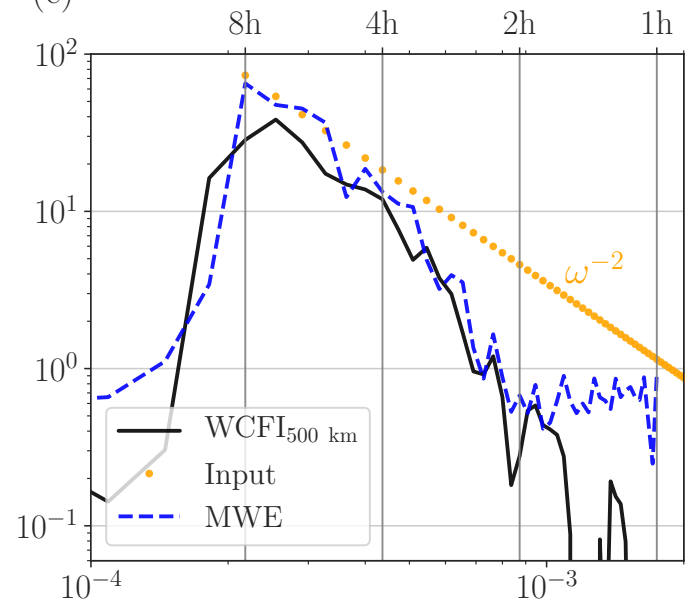

$\omega(\mathrm{rad} / \mathrm{s})$

Figure 4. (a): Contour plot shows the dependence of horizontal wavelength $\lambda_{h}$ with time-period $(T)$ and vertical wavelengths $\left(\lambda_{z}\right)$ of the simulated monochromatic GWs. (b): The orange dotted line shows the input frequency spectra with a power-law slope of -2 . The black and blue line plots illustrate the spectra estimated by the WCFI with $50 \mathrm{~km}$ horizontal resolution and MWE method, respectively using the GW model simulation. (c): The black line plot shows the WCFI spectra estimated with $500 \mathrm{~km}$ horizontal resolution. The blue and orange plots are identical to plot (b), which are added for comparison. 


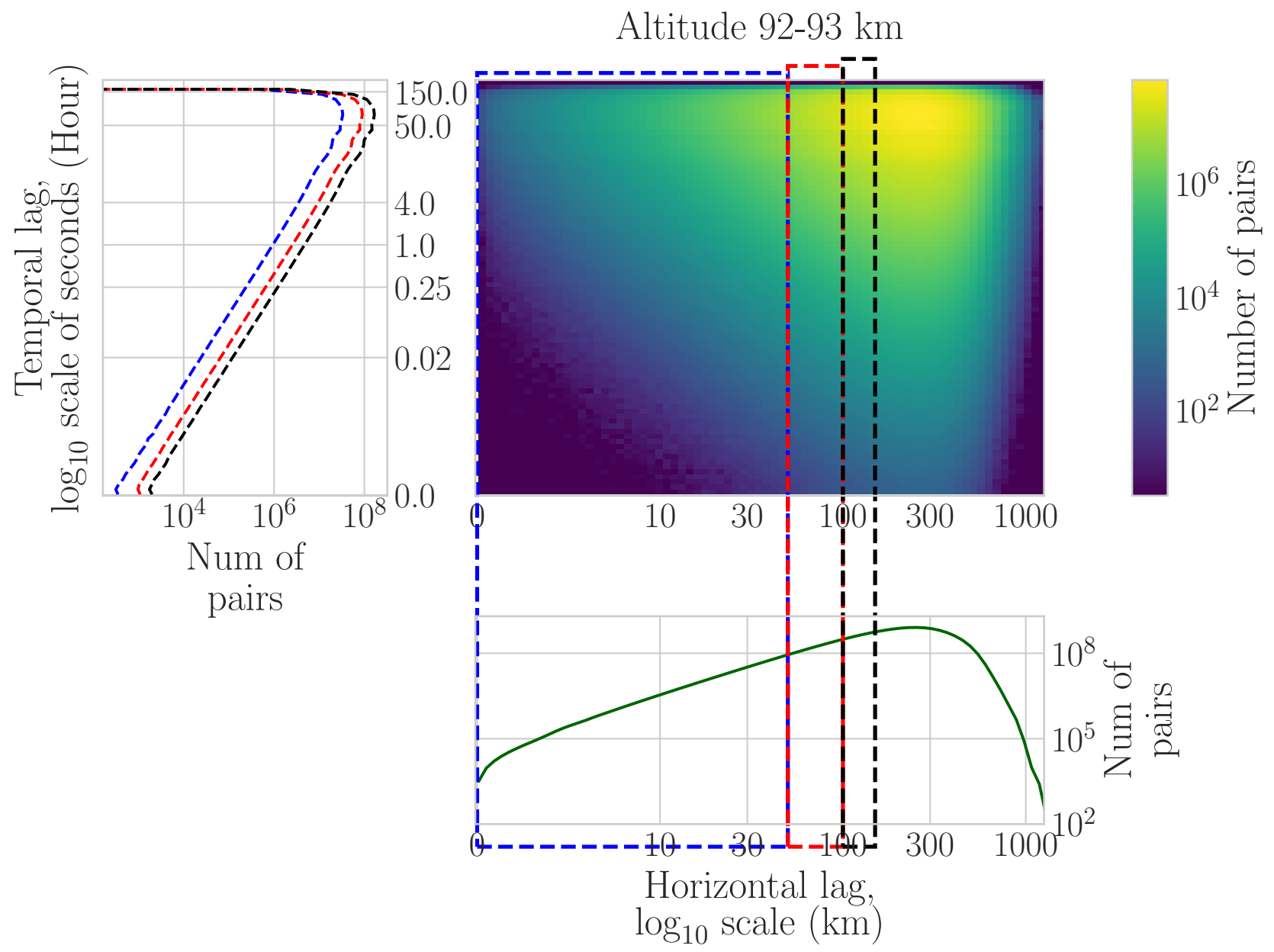

Figure 5. The two-dimensional histogram shows the measurement pairs between 92 and $93 \mathrm{~km}$ in altitude.

The black, red and, blue dashed boxes correspond to the meteor pairs used in three different horizontal scale analysis; 0-50 km, 50-100 km, 100-150 km respectively. The temporal lag histogram on the left shows the selected meteor pairs for the three different horizontal scales, where colours of dashed lines follow the dashed box colours in the 2D histogram. The horizontal lag histogram on the bottom shows the total meteor pairs averaged over all different temporal lags. 

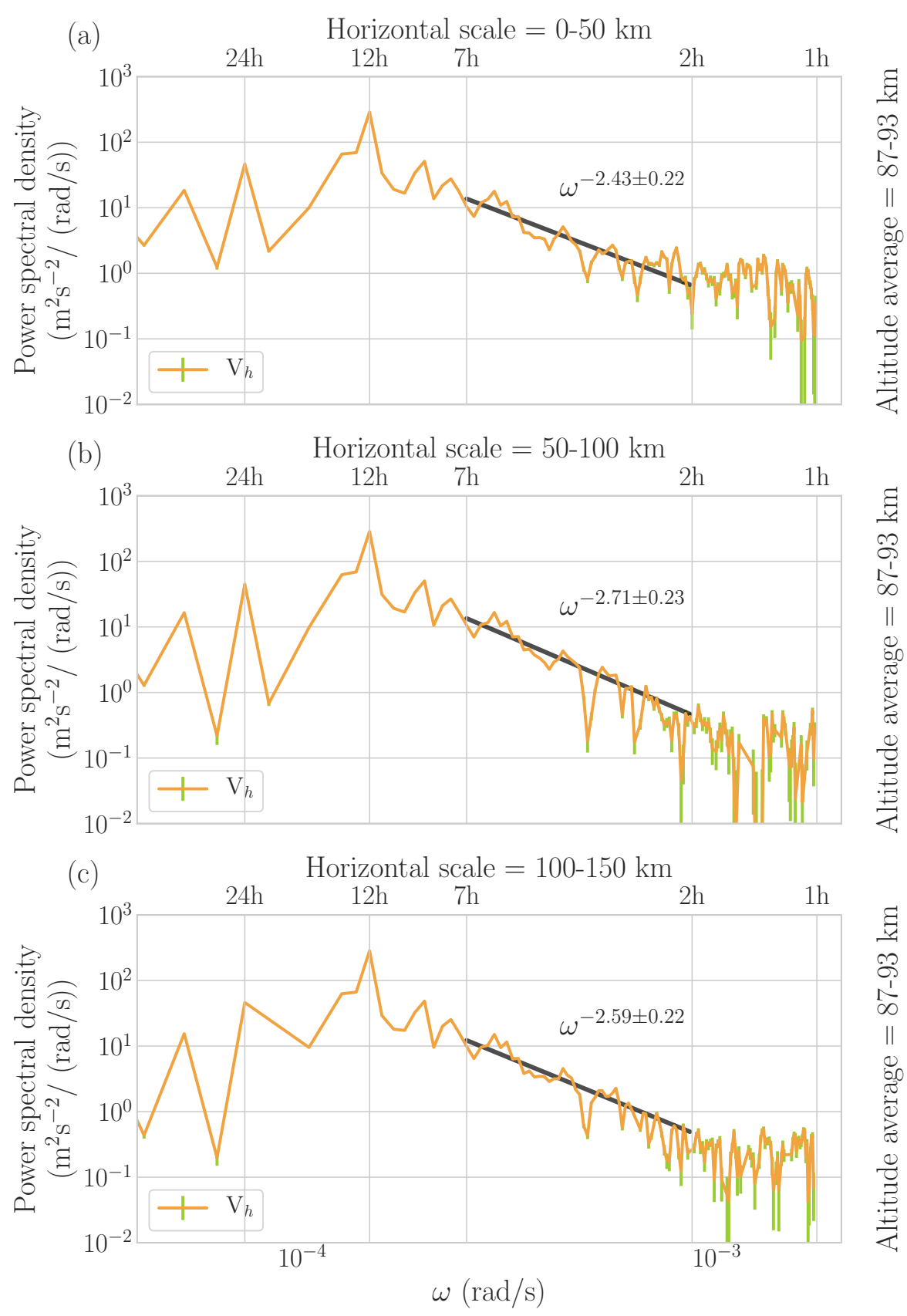

Figure 6. Plot (a), (b) and (c) show the horizontal frequency spectra estimated by WCFI method using three different horizontal scales: 0-50 km, 50-100 km and 100-150 km, respectively. The orange lines correspond to the frequency spectra and, the light green vertical lines show the standard error of spectra estimation on the spectra. All the spectra were estimated around $90( \pm 3) \mathrm{km}$ altitude. The solid black line on top of the spectra illustrates a power-law fit between ( $2 \pi / 7$ hour) and ( $2 \pi / 2$ hour). The slope values with standard error are inscribed in each plot. 

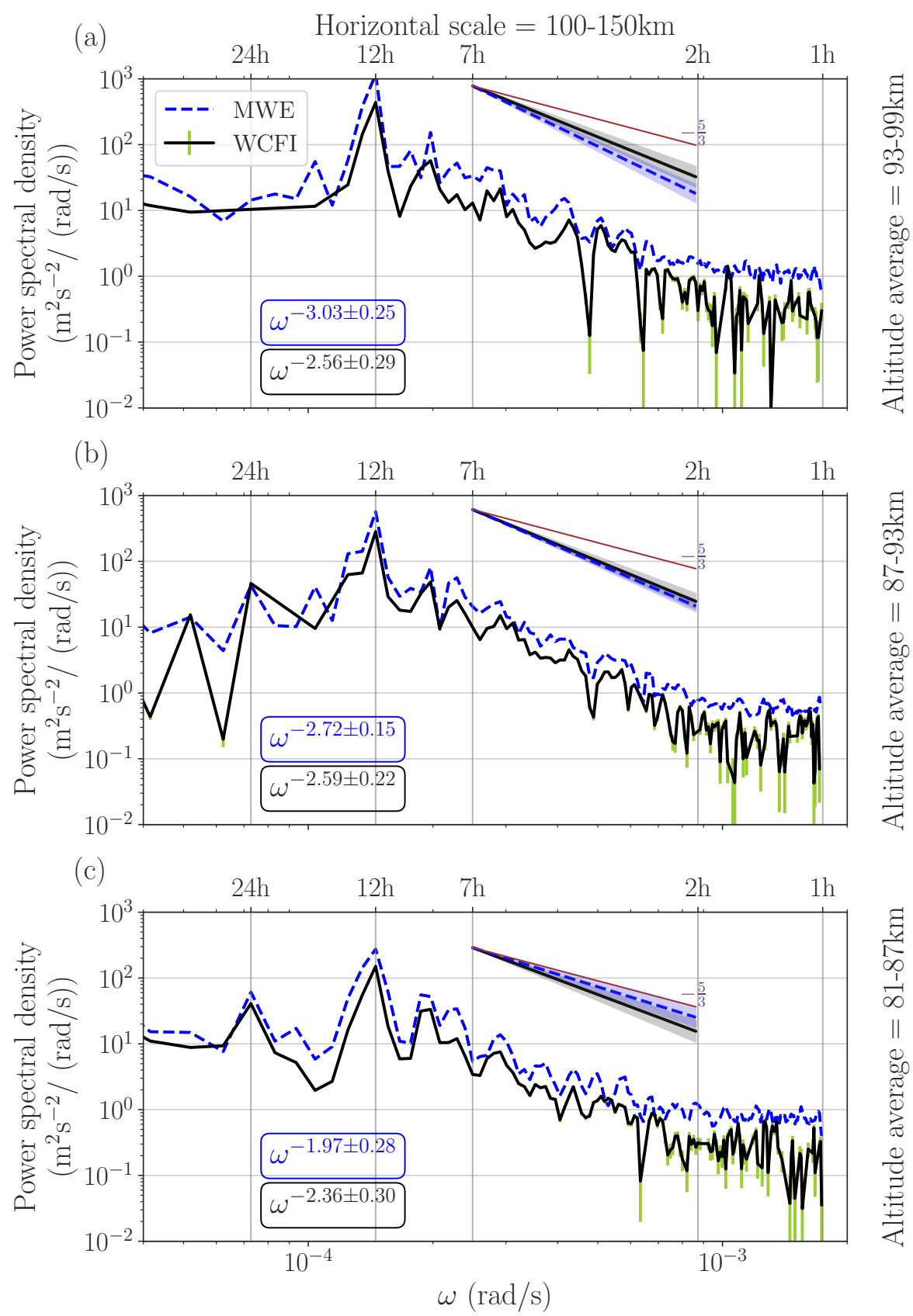

Figure 7. Plots (a), (b) and, (c) illustrate the frequency spectra of horizontal velocities obtained from the WCFI and MWE methods around three altitudes, i.e., $84( \pm 3), 90( \pm 3), 96( \pm 3) \mathrm{km}$. The solid black line in plots illustrate the spectra obtained from WCFI method, and the blue dashed lines in plots show the MWE spectra. The solid and transparent shades of black lines and blue lines in the plots show power-law slopes of the WCFI and MWE spectra, respectively. Slope values with standard error are inscribed in the plots in black and blue fonts which corresponds to WCFI and MWE spectra, respectively. 


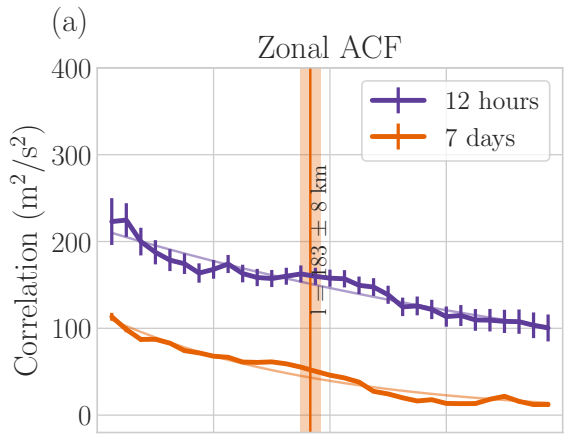

(c)

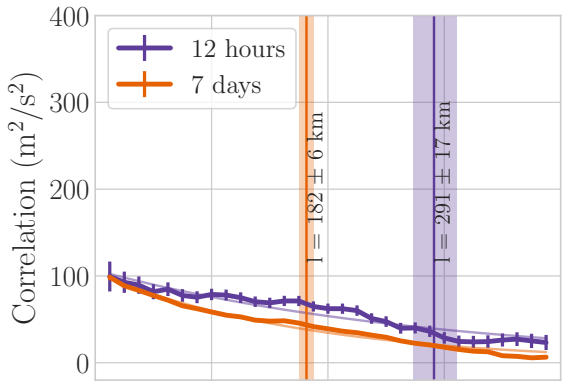

(e)

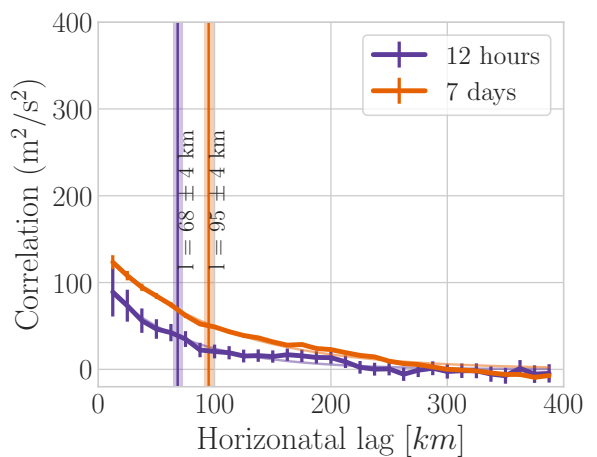

(b)

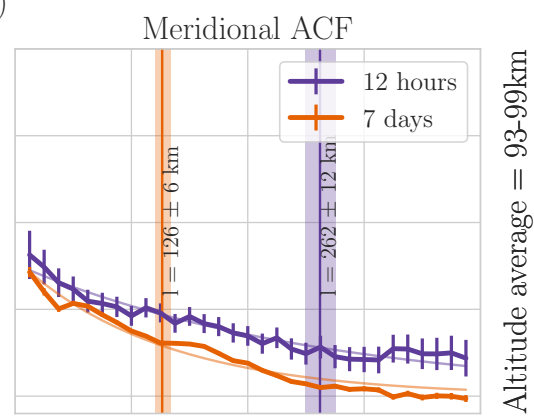

(d)

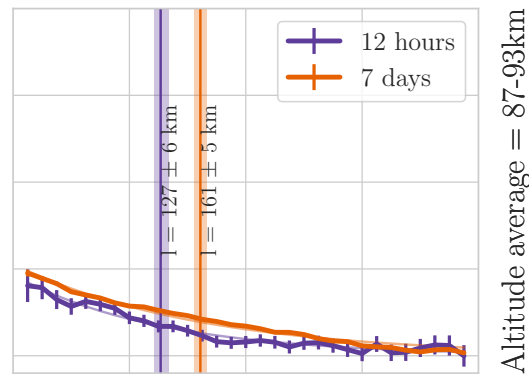

(f)

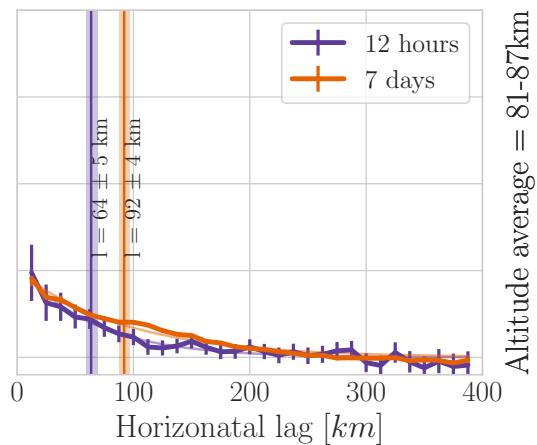

Figure 8. The orange line in the plots show the horizontal autocorrelation function (ACF) of four-hour high pass filtered zonal and meridional components using WCFI method during the seven days of the campaign. The purple lines show the horizontal ACFs of four-hour high pass filtered zonal and meridional wind components from a 12 hour during the campaign. Plots (a), (c) and, (e) show the ACFs of zonal components and plots (b), (d) and, (f) show the meridonal components averaged around $84( \pm 3), 90( \pm 3)$ and $96( \pm 3) \mathrm{km}$, respectively. Orange and purple vertical lines in the plots correspond to the e-folding length with their uncertainties of ACFs of 7 days and 12 hours, respectively. 
Table 1. Receiving sites for the SIMONe 2018 campaign. The name contains information about the frequency or the MIMO type to help the identification.

\begin{tabular}{|c|c|c|c|c|}
\hline Receiver & Latitude $\left(^{\circ}\right)$ & Longitude $\left(^{\circ}\right)$ & Transmission & Frequency $(\mathrm{MHz})$ \\
\hline Juliusruh & 54.63 & 13.37 & Pulsed & 32.55 \\
\hline Collm & 51.31 & 13.00 & Pulsed & 36.20 \\
\hline Bornim-32 & 52.44 & 13.02 & Pulsed & 32.55 \\
\hline Bornim-36 & 52.44 & 13.02 & Pulsed & 36.20 \\
\hline Neustrelitz-32 & 53.33 & 13.07 & Pulsed & 32.55 \\
\hline Neustrelitz-36 & 53.33 & 13.07 & Pulsed & 36.20 \\
\hline Bornim-MISO & 52.44 & 13.02 & coded-CW & 32.55 \\
\hline Bornim-SIMO & 52.44 & 13.02 & coded-CW & 32.55 \\
\hline Neustrelitz-MISO & 53.33 & 13.07 & coded-CW & 32.55 \\
\hline Neustrelitz-SIMO & 53.33 & 13.07 & coded-CW & 32.55 \\
\hline Breege-MISO & 54.62 & 13.37 & coded-CW & 32.55 \\
\hline Guderup-MISO & 55.00 & 9.86 & coded-CW & 32.55 \\
\hline Mechelsdorf-MISO & 54.12 & 11.67 & coded-CW & 32.55 \\
\hline Salzwedel-MISO & 52.75 & 10.90 & coded-CW & 32.55 \\
\hline
\end{tabular}




\section{Supplementary Files}

This is a list of supplementary files associated with this preprint. Click to download.

- graphicalabstract.png 